\title{
An Efficient Catalyst-Free Protocol for the Synthesis of Quinoxaline Derivatives under Ultrasound Irradiation
}

\author{
Wen-Xue Guo, ${ }^{a}$ Hui-Le Jin, ${ }^{a}$ Jiu-Xi Chen, ${ }^{*, a}$ Fan Chen, ${ }^{a}$ Jin-Chang Ding ${ }^{a, b}$ and Hua-Yue Wu*,a \\ ${ }^{a}$ College of Chemistry and Materials Engineering, Wenzhou University, Wenzhou, 325027, China \\ ${ }^{b}$ Wenzhou Vocational and Technical College, Wenzhou, 325035, China
}

\begin{abstract}
Um método altamente eficiente e simples foi descrito para a síntese de derivados de quinoxalina em bons a excelentes rendimentos (80-99\%) por reação de condensação de 1,2-dicetonas heterocíclicas ou alifáticas $\left(\mathrm{R}^{1} \mathrm{COCOR}^{1}, \mathrm{R}^{1}=\mathrm{Et}, \mathrm{Ph}, p-\mathrm{MeC}_{6} \mathrm{H}_{5}, p-\mathrm{MeOC}_{6} \mathrm{H}_{5}\right.$, Furil) com 1,2-diaminas (1,2-( $\left.\left.\mathrm{NH}_{2}\right)_{2} \mathrm{C}_{6} \mathrm{H}_{3} \mathrm{R}^{2}, \mathrm{R}^{2}=\mathrm{H}, \mathrm{Br}, \mathrm{NO}_{2}, \mathrm{PhCO}\right)$. Um estudo sistemático foi realizado para examinar a influência do meio reacional e dos fatores eletrônicos dos substratos nos resultados das reações.
\end{abstract}

A highly efficient and facile method has been described for the synthesis of quinoxaline derivatives in good to excellent yields (80-99\%) by condensation reaction of heterocyclic as well as aliphatic 1,2-diketones $\left(\mathrm{R}^{1} \mathrm{COCOR}{ }^{1}, \mathrm{R}^{1}=\mathrm{Et}, \mathrm{Ph}, p\right.$ - $\mathrm{MeC}_{6} \mathrm{H}_{5}, p$ - $\mathrm{MeOC}_{6} \mathrm{H}_{5}$, Furyl) with 1,2-diamines (1,2-( $\left.\left.\mathrm{NH}_{2}\right)_{2} \mathrm{C}_{6} \mathrm{H}_{3} \mathrm{R}^{2}, \mathrm{R}^{2}=\mathrm{H}, \mathrm{Br}, \mathrm{NO}_{2}, \mathrm{PhCO}\right)$. A systematic study was carried out to examine the influence of reaction media and electronic factors of the substrates on the reaction results.

Keywords: ultrasound irradiation, catalyst-free, quinoxaline derivatives

\section{Introduction}

Quinoxaline derivatives are well known in the pharmaceutical industry and have been shown to possess a broad spectrum of biological activities including antiviral, antibacterial, anti-inflammatory, askinase inhibitors, anticancer and anthelmintic agents. ${ }^{1}$ Quinoxaline ring is a part of a number of antibiotics such as echinomycin, levomycin, and actinomycin, which are known to inhibit the growth of Gram-positive bacteria and are also active against various transplantable tumors. ${ }^{2}$ Besides these, it has been reported for their application in dyes, ${ }^{3}$ efficient electroluminescent materials, ${ }^{4}$ organic semiconductors, ${ }^{5}$ building blocks for the synthesis of anion receptor, ${ }^{6}$ cavitands, ${ }^{7}$ dehydroannulenes, ${ }^{8}$ and DNA cleaving agents. ${ }^{9}$

In light of these significances, a variety of synthetic strategies have been developed for the preparation of quinoxaline derivatives. One of the most common methods is the condensation of 1,2-diamine with 1,2-dicarbonyl compounds in refluxing ethanol or acetic acid. ${ }^{10}$ Later, many improved methods has been reported for the

*e-mail: jiuxichen@wzu.edu.cn; huayuewu@wzu.edu.cn synthesis of various quinoxaline derivatives using the Bicatalyzed oxidative coupling, ${ }^{11}$ microwave irradiation, ${ }^{12}$ a solid-phase synthesis, ${ }^{13}$ and $\mathrm{RuCl}_{2}-\left(\mathrm{PPh}_{3}\right)_{3}$-TEMPO, ${ }^{14}$ $\mathrm{MnO}_{2},{ }^{15} \mathrm{POCl}_{3},{ }^{16}$ zeolites, ${ }^{17}$ iodine, ${ }^{18}$ cerium ammonium nitrate, ${ }^{19} \mathrm{CuSO}_{4} \cdot 5 \mathrm{H}_{2} \mathrm{O}^{20}$ and $\mathrm{SA} / \mathrm{MeOH},{ }^{21}$ Montmorillonite $\mathrm{K}-10,{ }^{22} \mathrm{Zn}[(\mathrm{L})$ proline $],{ }^{23}$ ionic liquid, ${ }^{24} \mathrm{Ni}$-nanoparticles, ${ }^{25}$ silica sulfuric acid, ${ }^{26} \mathrm{NH}_{4} \mathrm{Cl}^{27}$ as catalyst. Despite the progress, the state-of-the-art for the synthesis of these compounds remains less than ideal. Thus, the development of environmentally friendly benign, high-yielding and clean approaches for the synthesis of quinoxaline derivatives is still remains a highly desired goal in organic synthesis.

Ultrasonic-assisted organic synthesis as a green synthetic approach is a powerful technique that is being used more and more to accelerate organic reactions. ${ }^{28}$ Compared with traditional methods, this method is more convenient and reactions can be carried out in higher yield, shorter reaction time and milder conditions. ${ }^{29}$ As part of current studies on the development of green and efficient organic methodologies,$^{30}$ we herein report an efficient catalyst-free and practical method for the synthesis of quinoxaline derivatives under ultrasound irradiation at room temperature. 


\section{Results and Discussion}

Initial studies focused on the screening of the solvents, bases as well as catalyst loading with the reaction of benzil and $o$-phenylenediamine as the model reaction at room temperature. Efforts were directed towards the evaluation of the synthesis of quinoxalines by ultrasound irradiation. With the same substrates, the reaction in ethanol, using ultrasound irradiation at room temperature afforded the products in excellent yield (98\%) for $60 \mathrm{~min}$. In contrast, the poor yields were obtained when the reactions were carried in boiling ethanol or in ethanol at room temperature without the aid of ultrasound irradiation (Table 1, entry 4). Thus, the efficacy of various solvents was investigated in the model reaction using benzil with $o$-phenylenediamine under ultrasound irradiation at room temperature and the results are summarized in Table 1. We examined aprotic solvents (such as $\mathrm{CH}_{2} \mathrm{Cl}_{2}, \mathrm{CH}_{3} \mathrm{CN}$, THF, DMSO, DMF, EtOAc, 1,4-dioxane) and protic solvents (such as $\mathrm{H}_{2} \mathrm{O}, \mathrm{EtOH}$, and $\mathrm{MeOH}$ ). Almost all of the solvents (such as $\mathrm{CH}_{2} \mathrm{Cl}_{2}(93 \%)$, $\mathrm{CH}_{3} \mathrm{CN}(97 \%)$, THF (85\%), DMSO (92\%), DMF (84\%), EtOAc (97\%), and 1,4-dioxane (91\%)) with the exception of $\mathrm{H}_{2} \mathrm{O}$ (yield $42 \%$ ) afforded the desired products in good yields. The protic solvent EtOH (yield 98\%) came out as a superior solvent in this transformation.

Table 1. Effects of solvents on the reaction of benzil and $o$-phenylenediamine under ultrasound irradiation ${ }^{\mathrm{a}}$

\begin{tabular}{lcc} 
& Solvent & Yield / $(\%)^{\mathrm{b}}$ \\
\hline Entry & $\mathrm{CH}_{2} \mathrm{Cl}_{2}$ & 93 \\
2 & $\mathrm{THF}$ & 85 \\
3 & $\mathrm{DMF}$ & 84 \\
4 & $\mathrm{EtOH}$ & $98\left(42^{\mathrm{c}}, 19^{\mathrm{d}}\right)$ \\
5 & $\mathrm{MeOH}$ & 97 \\
6 & $\mathrm{CH}_{3} \mathrm{CN}$ & 97 \\
7 & $\mathrm{DMSO}$ & 92 \\
8 & 1,4-Dioxane & 91 \\
9 & EtOAc & 97 \\
10 & $\mathrm{H}_{2} \mathrm{O}$ & 42 \\
\hline
\end{tabular}

aAll reactions were performed at $1 \mathrm{mmol}$ scale in $2 \mathrm{~mL}$ of solvent. ${ }^{b}$ Isolated yields. ${ }^{c}$ The reaction was carried out in boiling ethanol without ultrasound irradiation for $2 \mathrm{~h}$. ${ }^{\mathrm{d}}$ The reaction was carried out in ethanol without ultrasound irradiation at room temperature for $3 \mathrm{~h}$.

With optimal conditions in hand, the reaction of various 1,2-diketones and with 1,2-diamines was examined to explore the scope of the reaction and the results are summarized in Table 2. The substitution groups on the aromatic ring associated with 1,2-diketone had no obvious effect on the yields. It was observed that electron-donating groups associated with aromatic 1,2-diketone decreased slightly the product yields (Table 2, entries 4-8). Moreover, we also examined the condensation of heterocyclic 1,2-diketone, such as 1,2-di(furan-2-yl)ethane-1,2-dione (Table 2, entries 9 and 10) with various 1,2-diamine. Similarly, the corresponding products $\mathbf{3 i}$ and $\mathbf{3} \mathbf{j}$ were obtained in excellent yield. When aliphatic 1,2-diketone, such as hexane-3,4-dione were used as reaction substrates, the desired products $\mathbf{3 k}$ and $\mathbf{3 1}$ were obtained in $80 \%$ and $81 \%$ yields, respectively (Table 2, entries 11 and 12). On the other hand, we investigated the influence of electronic factors of 1,2-diamine on the reaction results. It was observed that the reaction of 1,2-diamine bearing electron-donating group ( $-\mathrm{Me}$ ) on the benzene ring, such as 4-methylbenzene-1,2-diamine with various 1,2-diketones was examined and the corresponding products $\mathbf{3 b}, \mathbf{3 e}$ and 3h were obtained in good yields (Table 2, entries 2, 5 and 8). However, the presence of an electron-withdrawing group on the benzene ring decreased the reactivity of the substrate. For instance, 1,2-diamines containing strongly electron-withdrawing group $\left(-\mathrm{NO}_{2}\right)$ on the benzene ring, such as 4-nitrobenzene-1,2-diamine afforded the corresponding products $\mathbf{3 c}$ and $\mathbf{3 f}$ in only $37 \%$ and $36 \%$ yields, respectively (Table 2, entries 3 and 6), which showed an obvious electronic effect.

Table 2. Synthesis of quinoxaline derivatives under ultrasound irradiation using different diamines and 1,2-diketones ${ }^{\mathrm{a}}$

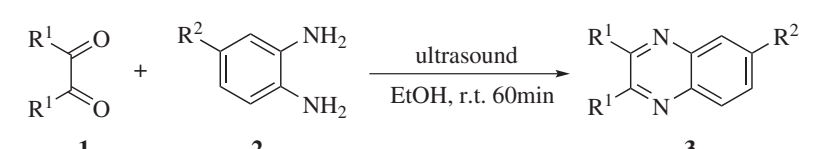

\begin{tabular}{lcccc}
\hline Entry & $\mathrm{R}^{1}$ & $\mathrm{R}^{2}$ & Product & Yield / $(\%)^{\mathrm{b}}$ \\
\hline 1 & $\mathrm{Ph}$ & $\mathrm{H}$ & $\mathbf{3 a}$ & 98 \\
2 & $\mathrm{Ph}$ & $\mathrm{Me}$ & $\mathbf{3 b}$ & 99 \\
3 & $\mathrm{Ph}$ & $\mathrm{NO}_{2}$ & $\mathbf{3 c}$ & 37 \\
4 & $p-\mathrm{MeC}_{6} \mathrm{H}_{4}$ & $\mathrm{H}$ & $\mathbf{3 d}$ & 93 \\
5 & $p-\mathrm{MeC}_{6} \mathrm{H}_{4}$ & $\mathrm{Me}$ & $\mathbf{3 e}$ & 95 \\
6 & $p-\mathrm{MeC}_{6} \mathrm{H}_{4}$ & $\mathrm{NO}_{2}$ & $\mathbf{3 f}$ & 36 \\
7 & $p-\mathrm{MeOC}_{6} \mathrm{H}_{4}$ & $\mathrm{H}$ & $\mathbf{3 g}$ & 90 \\
8 & $p-\mathrm{MeOC}_{6} \mathrm{H}_{4}$ & $\mathrm{Me}$ & $\mathbf{3 h}$ & 91 \\
9 & Furyl & $\mathrm{H}$ & $\mathbf{3 i}$ & 96 \\
10 & Furyl & $\mathrm{Me}$ & $\mathbf{3 j}$ & 96 \\
11 & Et & $\mathrm{H}$ & $\mathbf{3 k}$ & 81 \\
12 & Et & $\mathrm{Me}$ & $\mathbf{3 l}$ & 80 \\
\hline
\end{tabular}

${ }^{\mathrm{a}} \mathrm{All}$ reactions were performed at $0.5 \mathrm{mmol}$ scale in $2 \mathrm{~mL}$ of ethanol for $60 \mathrm{~min}$. 'Isolated yields. 
Thus, further optimization studies were performed in order to develop a better system that was more functional group compatible. The most dramatic improvement was observed when the solvent was switched from EtOH to EtOH/acetic acid (AcOH). AcOH does work not merely as a solvent, but also as a promoter of the reaction. Therefore, the volume ratio of $\mathrm{EtOH}$ and $\mathrm{AcOH}$ was examined and the best results were obtained by carrying out the reaction in $\mathrm{EtOH} / \mathrm{AcOH}$ with a ratio of 10:1 (v/v) (Table 3, entry 3).

Table 3. The influence of the amount of acetic acid ${ }^{\mathrm{a}}$

\begin{tabular}{lcc} 
Entry & $\mathrm{AcOH} / \mathrm{mL}$ & $\mathrm{Yield}^{\mathrm{b}} /(\%)$ \\
\hline 1 & - & 37 \\
2 & 0.1 & 85 \\
3 & 0.2 & 98 \\
4 & 0.3 & 98 \\
5 & 0.5 & 97 \\
\hline
\end{tabular}

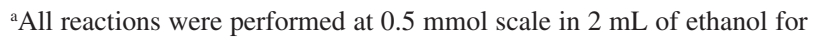
45 min. 'Isolated yields.

Next, we explored the scope of the condensation of 1,2-diketones with electron-withdrawing groups with 1,2-diamines (Table 4). In all the case, the reaction proceeded smoothly and the desired products were obtained in excellent yields.

To check the versatility of this method, the present protocol was also applied to less nucleophilic aromatic diamine such as naphthalene-2,3-diamine (Table 4, entry 4). On the other hand, some heterocyclic 1,2-diketone such as furil was subjected for condensation reaction and the desired products was obtained in excellent yields (Table 4, entries 6-7). When aliphatic 1,2-diketone, such as 3,4-hexanedione was used as substrate, the desired products were also obtained with excellent yield (Table 4, entry 8). Finally, we also examined the reaction of unsymmetrical 1,2-diketons with $o$-phenylenediamine (Table 4, entry 9). Similarly, the corresponding products were obtained with excellent yield.

In summary, we developed a highly efficient and facile method for the quinoxalines from various 1,2-diketones and 1,2-diamines under ultrasound irradiation at room temperature. Compared to previous reported methodologies, the present protocol features simple experimental operations, lower reaction temperature, high reaction rates and excellent yields, which makes this method a useful and attractive strategy in view of economic and environmental advantages.

Table 4. Synthesis of quinoxaline derivatives under ultrasound irradiation using different diamines and 1,2-diketones ${ }^{\mathrm{a}}$

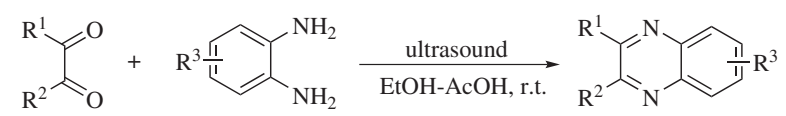

$\begin{array}{lll}1 & 2 & 3\end{array}$

\begin{tabular}{llcc}
\hline Entry & Product & Time $(\min )$ & Yield / $(\%)^{\mathrm{b}}$ \\
\hline 1 & & 45 & 98
\end{tabular}

2<smiles>Cc1ccc(-c2nc3ccc([N+](=O)[O-])cc3nc2-c2ccc(C)cc2)cc1</smiles>

3

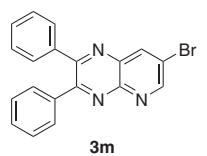

4

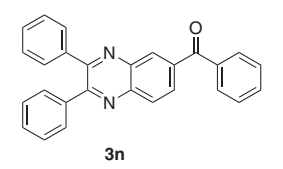

5

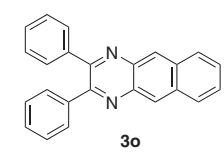

90

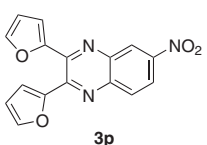

60

60

60

94

60

97

6

(1)

7

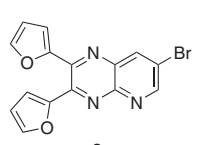

60

$3 q$

8

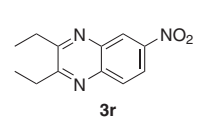

60

90

9

95

93

96

92

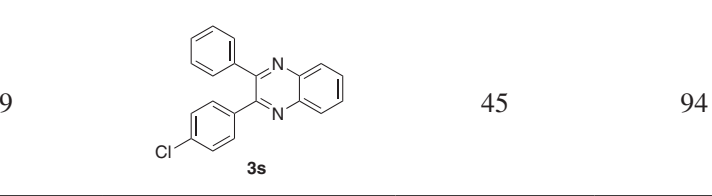

90

${ }^{\mathrm{a}}$ All reactions were performed at $0.5 \mathrm{mmol}$ scale in $2 \mathrm{~mL}$ of ethanol and $0.2 \mathrm{~mL}$ acetic acid. ${ }^{\text {IIsolated yields. }}$

\section{Experimental}

All reagents were commercial available and used without any purification. Melting points were recorded on Digital Melting Point Apparatus WRS-1B and are uncorrected. IR spectra were recorded on a BrukerEQUINOX55 spectrometer. ${ }^{1} \mathrm{H}$ NMR and ${ }^{13} \mathrm{C}$ NMR spectra 
were recorded on a Brucker AC 300 instrument using $\mathrm{CDCl}_{3}$ as the solvent with tetramethylsilane (TMS) as an internal standard at room temperature. Chemical shifts were given in $\delta$ relative to TMS, the coupling constants $J$ are given in $\mathrm{Hz}$. All reactions were conducted using standard Schlenk techniques. Column chromatography was performed using EM Silica gel 60 (300-400 mesh). Sonication was performed in Shanghai SY-5200DH water bath of the laboratory ultrasonic cleaner (with a frequency of $55 \mathrm{kHz}$ and a constant output power of 150W; Shanghai Shengyuan ultrasonic instrument Co., Ltd.). All reactions were carried out in Schlenk tubes under the air, and the Schlenk tube was located in the water bath of the ultrasonic cleaner, where the surface of reactants is slightly lower than the level of the water. The reaction temperature was controlled at $22-25^{\circ} \mathrm{C}$ by addition or removal of water from ultrasonic bath.

\section{General procedure for the preparation of quinoxalines 3}

A mixture of 1, 2-diketone (1, $0.5 \mathrm{mmol}), 1,2$-diamine (2, $0.5 \mathrm{mmol})$ and absolute ethanol $(2 \mathrm{~mL})$ or absolute ethanol/ acetic acid $(2 \mathrm{~mL} / 0.2 \mathrm{~mL})$ was irradiated under ultrasound in an open Schlenk tube at room temperature $\left(22-25^{\circ} \mathrm{C}\right)$ until completion of the reaction. The progress of the reaction was monitored by TLC. After the reaction was completed, the reaction mixture was concentrated in vacuo and the residue was purified by flash column chromatography (eluted with a mixture of petroleum ether and ethyl acetate) on a silica gel (300-400 mesh) to give the product 3 .

The spectral and analytical data of all compounds are given below.

\section{2,3-Diphenyl-quinoxaline $(3 \boldsymbol{a})^{18}$}

White solid, mp120-122 ${ }^{\circ} \mathrm{C},{ }^{1} \mathrm{H}$ NMR $\left(\mathrm{CDCl}_{3}\right.$, $300 \mathrm{MHz}): \delta 8.20(\mathrm{dd}, J 6.3,3.4 \mathrm{~Hz}, 2 \mathrm{H}), 7.76(\mathrm{dd}, J$ 6.3, $3.4 \mathrm{~Hz}, 2 \mathrm{H}), 7.53$ ca. 7.56 (m, 4H), 7.34 ca. 7.37 (m, 6H); ${ }^{13} \mathrm{C} \mathrm{NMR}\left(\mathrm{CDCl}_{3}, 75 \mathrm{MHz}\right): \delta$ 153.48, 141.29, 141.26, 141.24, 139.12, 130.03, 129.98, 129.94, 129.90, 129.87, $129.85,129.84,129.82,129.80,129.24,128.84,128.30$, 128.28, 128.26, 128.24; IR (KBr) $v_{\max } / \mathrm{cm}^{-1}: 3065,1441$, 1395, 1344, 768, 696.

\section{6-Methyl-2,3-diphenylquinoxaline $(\mathbf{3 b})^{18}$}

White solid, mp 135-137 ${ }^{\circ} \mathrm{C},{ }^{1} \mathrm{H}$ NMR $\left(\mathrm{CDCl}_{3}\right.$, $300 \mathrm{MHz}): \delta 8.07$ (d, J 8.5 Hz, 1H), $7.96(\mathrm{~s}, 1 \mathrm{H}), 7.50 \mathrm{ca}$. $7.53(\mathrm{~m}, 5 \mathrm{H}), 7.32 \mathrm{ca} .7 .35(\mathrm{~m}, 6 \mathrm{H}), 2.62(\mathrm{~s}, 3 \mathrm{H}) ;{ }^{13} \mathrm{C} \mathrm{NMR}$ $\left(\mathrm{CDCl}_{3}, 75 \mathrm{MHz}\right) \delta 153.00,152.26,140.98,140.16,139.40$, $138.92,131.97,130.59,129.52,129.02,128.94,128.39$, $128.36,128.30,127.90,127.71,127.31,126.89,126.83$, 21.59; IR (KBr) $v_{\max } / \mathrm{cm}^{-1}: 3053,1615,1488,1447,1341$, 670.

\section{6-Nitro-2,3-dipenylquinoxaline $(3 \boldsymbol{c})^{23}$}

White solid, mp 185-187 ${ }^{\circ} \mathrm{C},{ }^{1} \mathrm{H} \mathrm{NMR}\left(\mathrm{CDCl}_{3}\right.$, $300 \mathrm{MHz}): \delta 9.08$ (d, J 2.4 Hz, 1H), 8.53 (dd, J 9.1, $2.5 \mathrm{~Hz}$, $1 \mathrm{H}), 8.30(\mathrm{~d}, J 9.1 \mathrm{~Hz}, 1 \mathrm{H}), 7.55 \mathrm{ca} .7 .58(\mathrm{~m}, 4 \mathrm{H}), 7.35 \mathrm{ca}$. $7.44(\mathrm{~m}, 6 \mathrm{H}) ;{ }^{13} \mathrm{C} \mathrm{NMR}\left(\mathrm{CDCl}_{3}, 75 \mathrm{MHz}\right) \delta 143.35,139.74$, $137.86,137.79,130.75,130.66,130.52,129.67,129.59$, $129.54,129.41,129.26,128.60,128.52,128.23,127.21$, 127.12, 125.40, 124.25, 123.07; IR (KBr) v $\max / \mathrm{cm}^{-1}: 3439$, 1614, 1520, 1397, 1340, 699.

\section{2,3-Di-p-tolylquinoxaline $(\mathbf{3 d})^{18}$}

White solid, mp147-148 ${ }^{\circ} \mathrm{C},{ }^{1} \mathrm{H} \mathrm{NMR}\left(\mathrm{CDCl}_{3}\right.$, $300 \mathrm{MHz}): \delta 8.14$ ca. $8.15(\mathrm{~m}, 2 \mathrm{H}), 7.73$ ca. $7.76(\mathrm{~m}$, 2H), 7.44 (d, J 8.0, 4H), $7.16(\mathrm{~d}, J 7.9,4 \mathrm{H}), 2.38(\mathrm{~s}, 6 \mathrm{H})$; ${ }^{13} \mathrm{C}$ NMR $\left(\mathrm{CDCl}_{3}, 75 \mathrm{MHz}\right) \delta 153.17,140.83,138.44$, 136.06, 129.40, 129.35, 128.80, 128.68, 21.05; IR (KBr) $v_{\max } / \mathrm{cm}^{-1}: 2914,1608,1467,1397,1339,1217,759$.

\section{6-Methyl-2,3-di-p-tolylquinoxaline $(3 \boldsymbol{e})^{18}$}

White solid, mp135-136 ${ }^{\circ} \mathrm{C},{ }^{1} \mathrm{H} \mathrm{NMR}\left(\mathrm{CDCl}_{3}\right.$, $300 \mathrm{MHz}): \delta 8.03(\mathrm{~d}, J 8.5 \mathrm{~Hz}, 1 \mathrm{H}), 7.92(\mathrm{~s}, 1 \mathrm{H}), 7.58(\mathrm{~d}$, $J 1.8 \mathrm{~Hz}, 1 \mathrm{H}), 7.41$ (d, J 7.9 Hz, 4H), 7.14(d, J $8.0 \mathrm{~Hz}, 4 \mathrm{H})$, $2.60(\mathrm{~s}, 3 \mathrm{H}), 2.36(\mathrm{~s}, 6 \mathrm{H}) ;{ }^{13} \mathrm{C} \mathrm{NMR}\left(\mathrm{CDCl}_{3}, 75 \mathrm{MHz}\right) \delta$ $153.02,152.29,140.89,139.82,139.29,138.30,138.22$, 139.19, 131.69, 129.40, 128.64, $128.30127 .64,21.60$, 21.05; IR (KBr) $v_{\max } / \mathrm{cm}^{-1}: 3428,2913,1611,1448,1337$, 822 .

\section{6-Nitro-2,3-di-p-tolylquinoxaline $(\mathbf{3 f})^{18}$}

Yellow solid, mp 168-169 ${ }^{\circ} \mathrm{C},{ }^{1} \mathrm{H} \mathrm{NMR}\left(\mathrm{CDCl}_{3}\right.$, $300 \mathrm{MHz}): \delta 9.04(\mathrm{~d}, J 2.2 \mathrm{~Hz}, 1 \mathrm{H}), 8.49$ (dd, J 9.1, $2.3 \mathrm{~Hz}$, 1H), 8.25 (d, J 9.2 Hz, 1H), 7.45 ca. 7.49 (m, 4H), 7.18 $(\mathrm{d}, J 7.9,4 \mathrm{H}), 2.39$ (s ,6H); ${ }^{13} \mathrm{C} \mathrm{NMR}\left(\mathrm{CDCl}_{3}, 75 \mathrm{MHz}\right) \delta$ $156.29,155.67,143.53,140.00,139.81,135.36,130.58$, $129.79,129.69,129.16,125.52,123.00,21.42$; IR (KBr) $v_{\max } / \mathrm{cm}^{-1}: 3428,2918,1611,1523,1402,1343,828$.

\section{2,3-Bis(4-methoxyphenyl)quinoxaline $(\mathbf{3 g})^{18}$}

White solid, mp 146-148 ${ }^{\circ} \mathrm{C},{ }^{1} \mathrm{H}$ NMR $\left(\mathrm{CDCl}_{3}\right.$, $300 \mathrm{MHz}$ ): $\delta 8.13$ (dd, $J 6.3,3.4 \mathrm{~Hz}, 2 \mathrm{H}), 7.73$ (dd, $J$ 6.3, $3.4 \mathrm{~Hz}, 2 \mathrm{H}), 7.50$ (d, J 8.6 Hz, 4H), 6.88 (d, J 8.6Hz, 4H), $3.84(\mathrm{~s}, 6 \mathrm{H}) ;{ }^{13} \mathrm{C} \mathrm{NMR}\left(\mathrm{CDCl}_{3}, 75 \mathrm{MHz}\right) \delta 159.81,152.71$, 140.74, 131.38, 130.92, 129.23, 128.68, 113.45, 55.00; IR $(\mathrm{KBr}) v_{\max } / \mathrm{cm}^{-1}: 2930,2836,1605,1511,1344,1293$, $1246,1173,833$.

\section{2,3-Bis(4-methoxyphenyl)-6-methylquinoxaline $(\mathbf{3 h})^{18}$}

White solid, mp 121-123 ${ }^{\circ} \mathrm{C},{ }^{1} \mathrm{H} \mathrm{NMR}\left(\mathrm{CDCl}_{3}\right.$, $300 \mathrm{MHz}): \delta 8.00(\mathrm{~d}, J 8.2 \mathrm{~Hz}, 1 \mathrm{H}), 7.90(\mathrm{~s}, 1 \mathrm{H}), 7.46 \mathrm{ca}$. $7.56(\mathrm{~m}, 5 \mathrm{H}), 6.87$ (d, J 7.4 Hz, 4H), 3.83 (s, 6H), 2.59 (s, $3 \mathrm{H}) ;{ }^{13} \mathrm{C} \mathrm{NMR}\left(\mathrm{CDCl}_{3}, 75 \mathrm{MHz}\right) \delta 159.94,152.84,152.11$, 
$141.07,139.96,139.47,131.83,131.19,131.15,128.47$ 127.82, 113.68, 55.27, 21.86; IR (KBr) $v_{\max } / \mathrm{cm}^{-1}: 2925$, 2835, 1606, 1343, 1292, 1248, 1175, 833.

\section{2,3-Di-(furan-2-yl)quinoxaline $(3 i)^{18}$}

Pale brown solid, mp $131-132{ }^{\circ} \mathrm{C},{ }^{1} \mathrm{H}$ NMR $\left(\mathrm{CDCl}_{3}\right.$, $300 \mathrm{MHz}$ ): $\delta 8.12(\mathrm{dd}, J 6.3,3.4 \mathrm{~Hz}, 2 \mathrm{H}), 7.72$ (dd, $J 6.3$, $3.4 \mathrm{~Hz}, 2 \mathrm{H}), 7.61(\mathrm{~s}, 2 \mathrm{H}), 6.66(\mathrm{~d}, J 3.3 \mathrm{~Hz}, 2 \mathrm{H}), 6.55$ (s, $2 \mathrm{H}) ;{ }^{13} \mathrm{C}$ NMR $\left(\mathrm{CDCl}_{3}, 75 \mathrm{MHz}\right) \delta 150.74,144.13,142.53$, $140.53,130.30,129.02,112.93,111.85 ; \mathrm{IR}(\mathrm{KBr}) \mathrm{v}_{\max } / \mathrm{cm}^{-1}$ : 3103, 1566, 1484, 1397, 1328, 755.

\section{2,3-Di(furan-2-yl)-6-methylquinoxaline (3j) ${ }^{18}$}

Brown solid, mp $112-114{ }^{\circ} \mathrm{C},{ }^{1} \mathrm{H}$ NMR $\left(\mathrm{CDCl}_{3}\right.$, $300 \mathrm{MHz}): \delta 8.01(\mathrm{~d}, J 8.6 \mathrm{~Hz}, 1 \mathrm{H}), 7.91(\mathrm{~s}, 1 \mathrm{H}), 7.56$ ca. $7.61(\mathrm{~m}, 3 \mathrm{H}), 6.55$ ca. $6.62(\mathrm{~m}, 4 \mathrm{H}), 2.58(\mathrm{~s}, 3 \mathrm{H}) ;{ }^{13} \mathrm{C}$ NMR $\left(\mathrm{CDCl}_{3}, 75 \mathrm{MHz}\right) \delta 150.58,143.78,143.66,140.78$, $140.39,132.45,128.29,127.63,112.48,112.23,111.54$, 21.59; IR (KBr) $v_{\max } / \mathrm{cm}^{-1}: 3106,2918,1485,1323,747$.

\section{2,3-Diethylquinoxaline $(\mathbf{3 k})^{24}$}

Yellow oil, ${ }^{1} \mathrm{H}$ NMR $\left(\mathrm{CDCl}_{3}, 300 \mathrm{MHz}\right): \delta 7.99 \mathrm{ca} .8 .02$ (m, 2H), 7.62 ca. 7.65 (m, 2H), 3.03 (q, J 7.5 Hz, 4H), 1.41 (t, $J 4.5 \mathrm{~Hz}, 6 \mathrm{H}) ;{ }^{13} \mathrm{C}$ NMR $\left(\mathrm{CDCl}_{3}, 75 \mathrm{MHz}\right) \delta 156.87$; $140.65,128.28,128.12,28.00,12.20$; IR (KBr) $v_{\text {max }} / \mathrm{cm}^{-1}$ : 2972, 1709, 1451, 1286, 764 .

\section{2,3-Diethyl-6-methylquinoxaline $(3 l)^{24}$}

Pale yellow oil, ${ }^{1} \mathrm{H}$ NMR $\left(\mathrm{CDCl}_{3}, 300 \mathrm{M} \mathrm{Hz}\right): \delta 7.85$ $(\mathrm{d}, J 8.5,1 \mathrm{H}), 7.74(\mathrm{~s}, 1 \mathrm{H}), 7.43(\mathrm{~d}, J 8.5,1 \mathrm{H}), 2.98(\mathrm{dd}, J$ 14.9, $7.5 \mathrm{~Hz}, 4 \mathrm{H}), 2.51(\mathrm{~s}, 3 \mathrm{H}), 1.37(\mathrm{t}, J 7.5 \mathrm{~Hz}, 6 \mathrm{H}) ;{ }^{13} \mathrm{C}$ NMR $\left(\mathrm{CDCl}_{3}, 75 \mathrm{MHz}\right) \delta 156.72,155.90,138.56,130.49$, 127.59, 127.05, 27.96, 27.91, 21.33, 12.32, 12.28; IR (KBr) $v_{\max } / \mathrm{cm}^{-1}: 2971,1622,1565,1453,821$.

\section{7-Bromo-2,3-diphenylpyrido[3,2-b]pyrazine (3m $)^{31}$}

Pale yellow solid, mp $154-155^{\circ} \mathrm{C},{ }^{1} \mathrm{H}$ NMR $\left(\mathrm{CDCl}_{3}\right.$, $300 \mathrm{MHz}): \delta 9.08(\mathrm{~d}, J 1.4 \mathrm{~Hz}, 1 \mathrm{H}), 8.58(\mathrm{t}, J-1.0 \mathrm{~Hz}, 1 \mathrm{H})$, 7.48 ca. $7.58(\mathrm{~m}, 4 \mathrm{H}), 7.24$ ca. $7.32(\mathrm{~m}, 6 \mathrm{H}) ;{ }^{13} \mathrm{C}$ NMR $\left(\mathrm{CDCl}_{3}, 75 \mathrm{MHz}\right) \delta 156.17,155.14,154.77,139.04,137.77$, 137.47, 129.87, 129.50, 129.35, 129.26, 128.12, 127.91; IR $(\mathrm{KBr}) v_{\max } / \mathrm{cm}^{-1}: 3396,3057,1393,1331,697$.

\section{(2,3-Diphenylquinoxalin-6-yl)(phenyl)methanone $(\mathbf{3 n})^{18}$}

White solid, mp $139-140^{\circ} \mathrm{C},{ }^{1} \mathrm{H} \mathrm{NMR}\left(\mathrm{CDCl}_{3}, 300 \mathrm{MHz}\right)$ : $\delta 8.54(\mathrm{~s}, 1 \mathrm{H}), 8.28(\mathrm{~s}, 2 \mathrm{H}), 7.91(\mathrm{~d}, J 7.4 \mathrm{~Hz}, 2 \mathrm{H}), 7.63(\mathrm{~d}, J$ $7.3 \mathrm{~Hz}, 1 \mathrm{H}), 7.51 \mathrm{ca} .7 .57(\mathrm{~m}, 6 \mathrm{H}), 7.34 c a .7 .40(\mathrm{~m}, 6 \mathrm{H}) ;{ }^{13} \mathrm{C}$ NMR $\left(\mathrm{CDCl}_{3}, 75 \mathrm{MHz}\right) \delta 195.74,155.12,154.58,142.96$, 140.18, 138.63, 138.30, 137.20, 132.41, 130.09, 129.80, $129.74,129.25,129.11,128.48,128.33 ; \mathrm{IR}(\mathrm{KBr}) \mathrm{v}_{\max } / \mathrm{cm}^{-1}$ : 3432, 3052, 1661, 1438, 1396, 1344, 1270, 698.

\section{2,3-Diphenyl-[g]quinoxaline $(\mathbf{3 o})^{18}$}

Yellow solid, mp $188-189^{\circ} \mathrm{C},{ }^{1} \mathrm{H}$ NMR $\left(\mathrm{CDCl}_{3}\right.$, $300 \mathrm{MHz}$ ): $\delta$ 8.75.(s, 2H), $8.10 \mathrm{ca} .8 .13$ (m, 2H), $7.55 \mathrm{ca}$. $7.60(\mathrm{~m}, 6 \mathrm{H}), 7.36 \mathrm{ca} .7 .40(\mathrm{~m}, 6 \mathrm{H}) ;{ }^{13} \mathrm{C} \mathrm{NMR}\left(\mathrm{CDCl}_{3}\right.$, $75 \mathrm{MHz}) \delta 153.87,138.84,137.64,133.75,130.59,129.52$, 129.06, 128.96, 128.69, 128.45, 128.23, 127.93, 127.53, 127.24, 126.91, 126.41, 126.18, 125.29; IR (KBr) $v_{\max } / \mathrm{cm}^{-1}$. 3421, 3051, 1439, 1344, 1257, 1170, 695.

\section{2,3-Di(furan-2-yl)-6-nitroquinoxaline $(3 \boldsymbol{p})^{32}$}

Orange solid, mp $164-166{ }^{\circ} \mathrm{C},{ }^{1} \mathrm{H}$ NMR $\left(\mathrm{CDCl}_{3}\right.$, $300 \mathrm{MHz}): \delta 8.98$ (d, $J 2.4 \mathrm{~Hz}, 1 \mathrm{H}), 8.47$ (dd, $J 9.2,2.5 \mathrm{~Hz}$, 1H), $8.20(\mathrm{~d}, J 9.2 \mathrm{~Hz}, 1 \mathrm{H}), 7.65$ ca. $7.68(\mathrm{~m}, 2 \mathrm{H}), 6.85$ (dd, J 16.4, 3.5 Hz, 2H), $6.60 \mathrm{ca} .6 .63(\mathrm{~m}, 2 \mathrm{H}) ;{ }^{13} \mathrm{C} \mathrm{NMR}$ $\left(\mathrm{CDCl}_{3}, 75 \mathrm{MHz}\right) \delta 149.81,149.75,147.57,145.14,144.66$, 144.34, 143.84, 142.63, 138.87, 130.10, 124.99, 123.99, $123.28,115.04,114.17,112.10,111.96$; IR (KBr) $v_{\max } /$ $\mathrm{cm}^{-1}: 3388,1574,1522,1477,1337,749$.

\section{7-Bromo-2,3-(furan-2-yl)pyrido[3,2-b]pyrazine $(3 q)^{33}$}

Dark brown solid, mp134-136 ${ }^{\circ} \mathrm{C},{ }^{1} \mathrm{H}$ NMR $\left(\mathrm{CDCl}_{3}\right.$, $300 \mathrm{MHz}): \delta 9.05(\mathrm{~d}, J 2.4 \mathrm{~Hz}, 1 \mathrm{H}), 8.56(\mathrm{~d}, J 2.4 \mathrm{~Hz}$, 1H), $7.58 \mathrm{ca} .7 .62(\mathrm{~m}, 2 \mathrm{H}), 7.04(\mathrm{~d}, J 3.5 \mathrm{~Hz}, 1 \mathrm{H}), 6.76$ $(\mathrm{d}, J 3.5 \mathrm{~Hz}, 1 \mathrm{H}), 6.55$ ca. $6.59(\mathrm{~m}, 2 \mathrm{H}) ;{ }^{13} \mathrm{C} \mathrm{NMR}\left(\mathrm{CDCl}_{3}\right.$, $75 \mathrm{MHz}) \delta 154.92,150.00,149.77,147.16,144.76,144.66$, 143.43, 138.63, 135.64, 120.77, 114.76, 114.36, 112.08, 111.86; IR (KBr) $v_{\text {max }} / \mathrm{cm}^{-1}: 3112,1570,1479,1413,1322$, 758.

\section{2,3-Diethyl-6-nitroquinoxaline $(3 \boldsymbol{r})^{33}$}

Pale yellow solid, mp $98-100{ }^{\circ} \mathrm{C},{ }^{1} \mathrm{H}$ NMR $\left(\mathrm{CDCl}_{3}\right.$, $300 \mathrm{MHz}): \delta 8.78(\mathrm{~d}, J 2.5 \mathrm{~Hz}, 1 \mathrm{H}), 8.31(\mathrm{dd}, J 9.1,2.5 \mathrm{~Hz}$, $1 \mathrm{H}), 8.03(\mathrm{~d}, J 9.1 \mathrm{~Hz}, 1 \mathrm{H}), 3.04(\mathrm{q}, J 7.4 \mathrm{~Hz}, 4 \mathrm{H}), 1.41$ (t, $J$ 7.4, 6H); ${ }^{13} \mathrm{C} \mathrm{NMR}\left(\mathrm{CDCl}_{3}, 75 \mathrm{MHz}\right) \delta 160.42,159.42$, $146.51,143.19,139.30,129.65,124.60,121.60,28.13$, 27.91, 11.30, 11.24; IR (KBr) $v_{\max } / \mathrm{cm}^{-1}: 3430,2976,1614$, $1525,1339,1275,739$.

\section{2-(4-Chlorophenyl)-3-phenylquinoxaline $(3 s)^{34}$}

White solid, mp140-142 ${ }^{\circ} \mathrm{C},{ }^{1} \mathrm{H}$ NMR $\left(\mathrm{CDCl}_{3}\right.$, $300 \mathrm{MHz}): \delta 8.16 c a .8 .20(\mathrm{~m}, 2 \mathrm{H}), 7.78 c a .7 .81(\mathrm{~m}$, 2H), 7.47 ca. 7.53 (m, 4H), 7.31 ca. $7.39(\mathrm{~m}, 5 \mathrm{H}) ;{ }^{13} \mathrm{C}$ NMR $\left(\mathrm{CDCl}_{3}, 75 \mathrm{MHz}\right) \delta 140.96,140.86,138.49,137.17$, 134.79, 130.91, 129.89, 129.83, 129.46, 128.91, 128.85, 128.68, 128.22, 128.16; IR (KBr) $v_{\text {max }} / \mathrm{cm}^{-1}: 3429,3054$, 1590, 1484, 1396, 1341, 806.

\section{Acknowledgments}

We are grateful to the National Key Technology R\&D Program (No. 2007BAI34B00) and the Natural Science 
Foundation of Zhejiang Province (No. Y4080107) for financial support.

\section{Supplementary Information}

Supplementary data are available free of charge at http:// jbcs.sbq.org.br, as PDF file.

\section{References}

1. Sakata, G.; Makino, K.; Kuraswa, Y.; Heterocycles 1988, 27, 2481; He, W.; Meyers, M. R.; Hanney, B.; Spada, A.; Blider, G.; Galzeinski, H.; Amin, D.; Needle, S.; Page, K.; Jayyosi, Z.; Perrone, H.; Bioorg. Med. Chem. Lett. 2003, 13, 3097; Kim, Y. B.; Kim, Y. H.; Park, J. Y.; Kim, S. K.; Bioorg. Med. Chem. Lett. 2004, 14, 541.

2. Dell, A.; William, D. H.; Morris, H. R.; Smith, G. A.; Feeney, J.; Roberts, G. C. K.; J. Am. Chem. Soc. 1975, 97, 2497; Bailly, C.; Echepare, S.; Gago, F.; Waring, M.; Anti-Cancer Drug Des. 1999, 15, 291; Sato, S.; Shiratori, O.; Katagiri, K. J.; Antibiot. 1967, 20, 270.

3. Brock, E. D.; Lewis, D. M.; Yousaf, T. I.; Harper, H. H.; The Procter and Gamble Company: USA, WO 9951688, 1999.

4. Justin Thomas, K. R.; Marappan, V.; Jiann, T. L.; Chang-Hao, C.; Yu-ai, T.; Chem. Mater. 2005, 17, 1860.

5. Dailey, S.; Feast, J. W.; Peace, R. J.; Saga, R. C.; Till, S.; Wood, E. L.; J. Mater. Chem. 2001, 11, 2238; Brien, D. O.; Weaver, M. S.; Lidzey, D. G.; Bradley, D. D. C.; Appl. Phys. Lett.. 1996, 69,881 .

6. Jonathan, L. S.; Hiromitsu, M.; Toshihisa, M.; Vincent, M. L.; Hiroyuki, F.; Chem. Commun. 2002, 862.

7. Jonathan, L. S.; Hiromitsu, M.; Toshihisa, M.; Vincent, M. L.; Hiroyuki, F.; J. Am. Chem. Soc. 2002, 124, 13474; Peter, P. C.; Gang, Z.; Grace, A. M.; Carlos, H.; Linda, M. G. T.; Org. Lett. 2004, 6, 333.

8. Sascha, O.; Rudiger, F.; Synlett 2004, 1509.

9. Louis, S.; Marc, M. G.; Jory, J. W.; Joseph, P. B.; J. Org. Chem. 2003, 68, 4179.

10. Porter, A. E. A. In Comprehensive Heterocyclic Chemistry; Katritsky, A. R.; Rees, C. W.; eds.; Pergamon: Oxford, 1984, 157; Woo, G. H. C.; Snyder, J. K.; Wan, Z. K.; Prog. Heterocycl. Chem. 2002, 14, 279; Brown, D. J. In The Chemistry of Heterocyclic Compounds; Taylor, E. C.; Wipf, P., eds.; John Wiley\& Sons: New Jersey, 2004.

11. Antoniotti, S.; Donach, E.; Tetrahedron Lett. 2002, 43, 3971.

12. Zhao, Z.; Wisnoski, D. D.; Wolkenberg, S. E.; Leister, W. H.; Wang, Y.; Lindsley, C. W.; Tetrahedron Lett. 2004, 45, 4873; Gris, J.; Glisoni, R.; Fabian, L.; Fernández, B.; Moglioni, A. G.; Tetrahedron Lett. 2008, 49, 1053; Mohsenzadeh, F.; Aghapoor, K.; Darabi, H. R.; J. Braz. Chem. Soc. 2007, 18, 297.

13. Wu, Z.; Ede, N. J.; Tetrahedron Lett. 2001, 42, 8115.
14. Robinson, R. S.; Taylor, R. J. K.; Synlett 2005, 1003.

15. Raw, S. A.; Wilfred, C. D.; Taylor, R. J. K.; Org. Biomol. Chem. 2004, 2, 788.

16. Venkatesh, C.; Singh, B.; Mahata, P. K.; IIa, H.; Junjappa, H.; Org. Lett. 2005, 7, 2169.

17. Bhosale, R. S.; Sarda, S. R.; Ardhapure, S. S.; Jadhav, W. N.; Bhusare, S. R.; Pawar, R. P.; Tetrahedron Lett. 2005, 46, 7183.

18. Steven, A. R.; Cecilia, D. W.; Richard, J. K. T.; Chem. Commun. 2003, 2286; Bhosale, R. S.; Sarda, S. R.; Ardhapure, S. S.; Jadhav, W. N.; Bhusare, S. R.; Pawar, R. P.; Tetrahedron Lett. 2005, 46, 7183; More, S. V.; Sastry, M. N. V.; Wang, C. C.; Yao, C. F.; Tetrahedron Lett. 2005, 46, 6345.

19. More, S. V.; Sastry, M. N. V.; Yao, C. F.; Green Chem. 2006, 8, 91.

20. Heravi, M. M.; Taheri, S.; Bakhtiari, K.; Oskooie, H. A.; Catal. Commun. 2007, 8, 211.

21. Darabi, H. R.; Mohandessi, S.; Aghapoor, K.; Mohsenzadeh, F.; Catal. Commun. 2007, 8, 389.

22. Huang, T. K.; Wang, R.; Shi, L.; Lu, X. X.; Catal. Commun. 2008, 9, 1143.

23. Heravi, M. M.; Tehrani, M. H.; Bakhtiari, K.; Oskooie, H. A.; Catal. Commun. 2007, 8, 1341.

24. Fang, D.; Gong, K.; Fei, Z.; Zhou, X.; Liu, Z.; Catal. Commun. 2008, 9, 317; Potewar, T. M.; Ingale, S. A.; Srinivasan, K. V.; Synth. Commun. 2008, 38, 3601.

25. Kumar, A.; Kumar, S.; Saxena, A.; De, A.; Mozumdar, S.; Catal. Commun. 2008, 9, 778.

26. Shaabani, A.; Maleki, A.; Chin. J. Chem. 2007, 25, 818.

27. Darabi, H. R.; Tahoori, F.; Aghapoor, K.; Taala, F.; Mohsenzadeh, F.; J. Braz. Chem. Soc. 2008, 19, 1646.

28. Xu, H.; Liao, W. M.; Li, H. F.; Ultrason. Sonochem. 2007, 14, 779.

29. Mason, T. J.; Peters, D.; Practical Sonochemistry, Power Ultrasound Uses and Applications, $2^{\text {nd }}$ ed., Ellis Horwood Publishers: Chicherster, 2002.

30. Chen, J. X.; Su, W. K.; Wu, H. Y.; Liu, M. C.; Jin, C.; Green Chem. 2007, 9, 972; Xiong, W.; Chen, J. X.; Liu, M. C.; Ding, J. C.; Wu, H. Y. Su, W. K.; J. Braz. Chem. Soc. 2009, 20, 367 and references cited therein.

31. Yin, L. X.; Liebscher, J.; Synthesis 2005, 8, 1345.

32. Srinivas, C.; Kumar, C. N. S. S. P.; Rao, V. J.; Palaniappan, S.; J. Mol. Catal. A: Chem. 2007, 265, 227.

33. Kawakami, J.; Duncton, M.; Sherman, D.; He, H. Y.; Kiselyov, A.; Pytowski, B.; WO pat. 2,005,007,099 2005. (CA 142:176856).

34. Ebisawa, A.; Inoue, T.; Jpn. Kokai Tokkyo Koho 09,188,874 1997. (CA 127:142616).

Received: February 12, 2009 Web Release Date: September 11, 2009 


\section{An Efficient Catalyst-Free Protocol for the Synthesis of Quinoxaline Derivatives under Ultrasound Irradiation}

Wen-Xue Guo, ${ }^{a}$ Hui-Le Jin, ${ }^{a}$ Jiu-Xi Chen, ${ }^{*, a}$ Fan Chen, ${ }^{a}$ Jin-Chang Ding ${ }^{a, b}$ and Hua-Yue Wu ${ }^{*, a}$

${ }^{a}$ College of Chemistry and Materials Engineering, Wenzhou University, Wenzhou, 325027, China

${ }^{b}$ Wenzhou Vocational and Technical College, Wenzhou, 325035, China

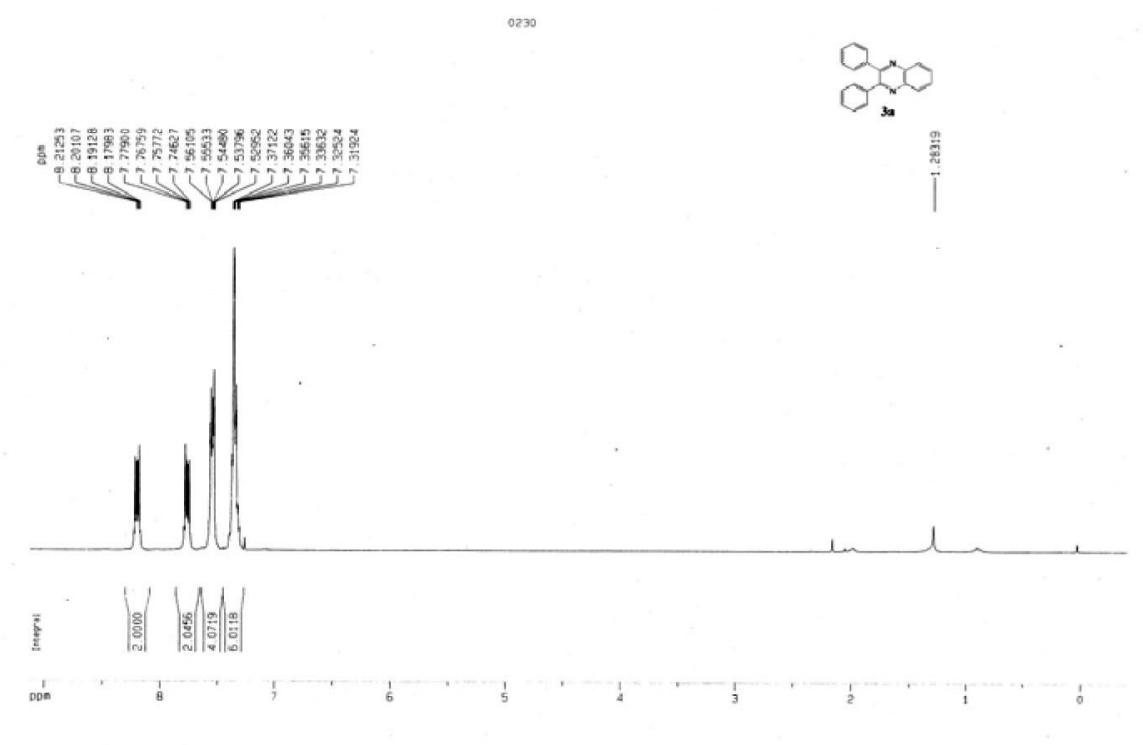

0230

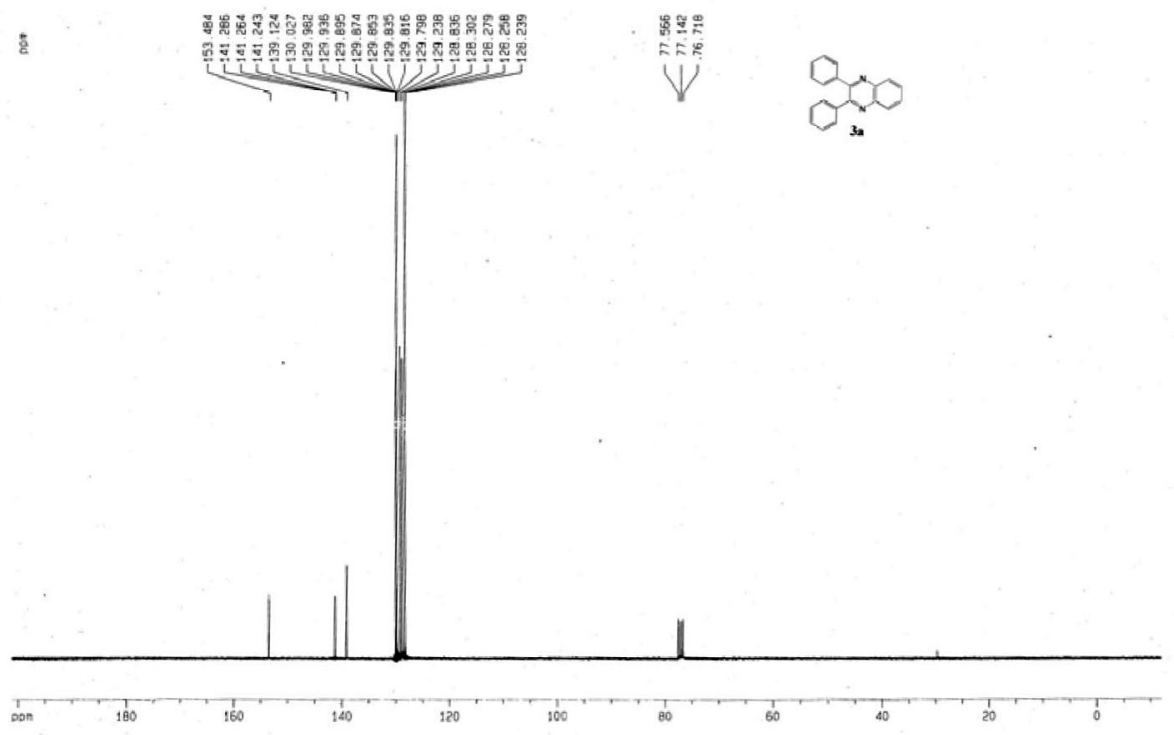

Figure S1. ${ }^{1} \mathrm{H}$ NMR of 3a (300 MHz, $\left.\mathrm{CDCl}_{3}\right)$ and ${ }^{13} \mathrm{C} \mathrm{NMR}$ of $\mathbf{3 a}\left(75 \mathrm{MHz}, \mathrm{CDCl}_{3}\right)$.

*e-mail: jiuxichen@wzu.edu.cn; huayuewu@wzu.edu.cn 


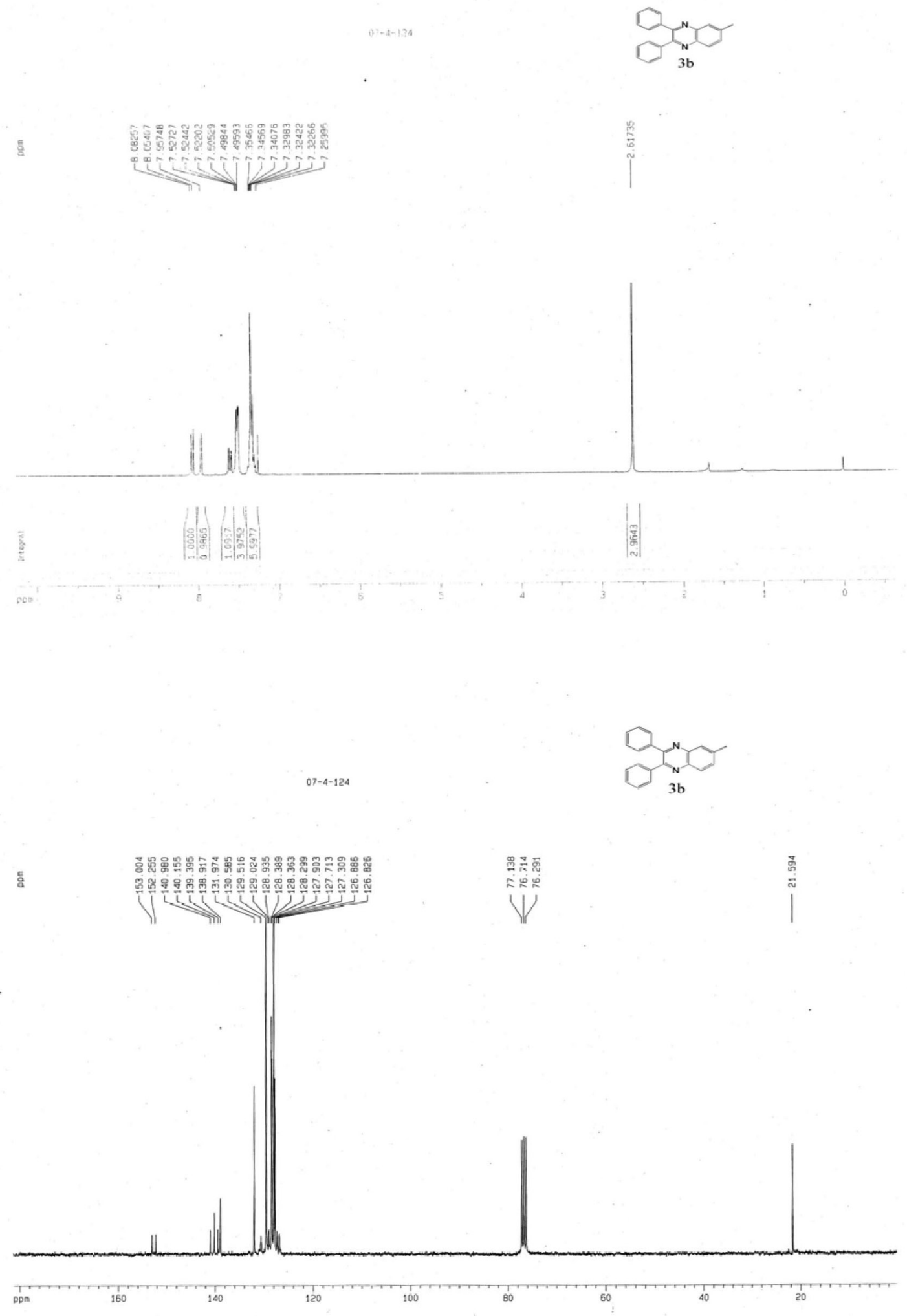

Figure S2. ${ }^{1} \mathrm{H}$ NMR of $\mathbf{3 b}\left(300 \mathrm{MHz}, \mathrm{CDCl}_{3}\right)$ and ${ }^{13} \mathrm{C} \mathrm{NMR}$ of $\mathbf{3 b}\left(75 \mathrm{MHz}, \mathrm{CDCl}_{3}\right)$. 


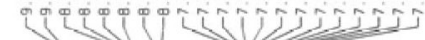
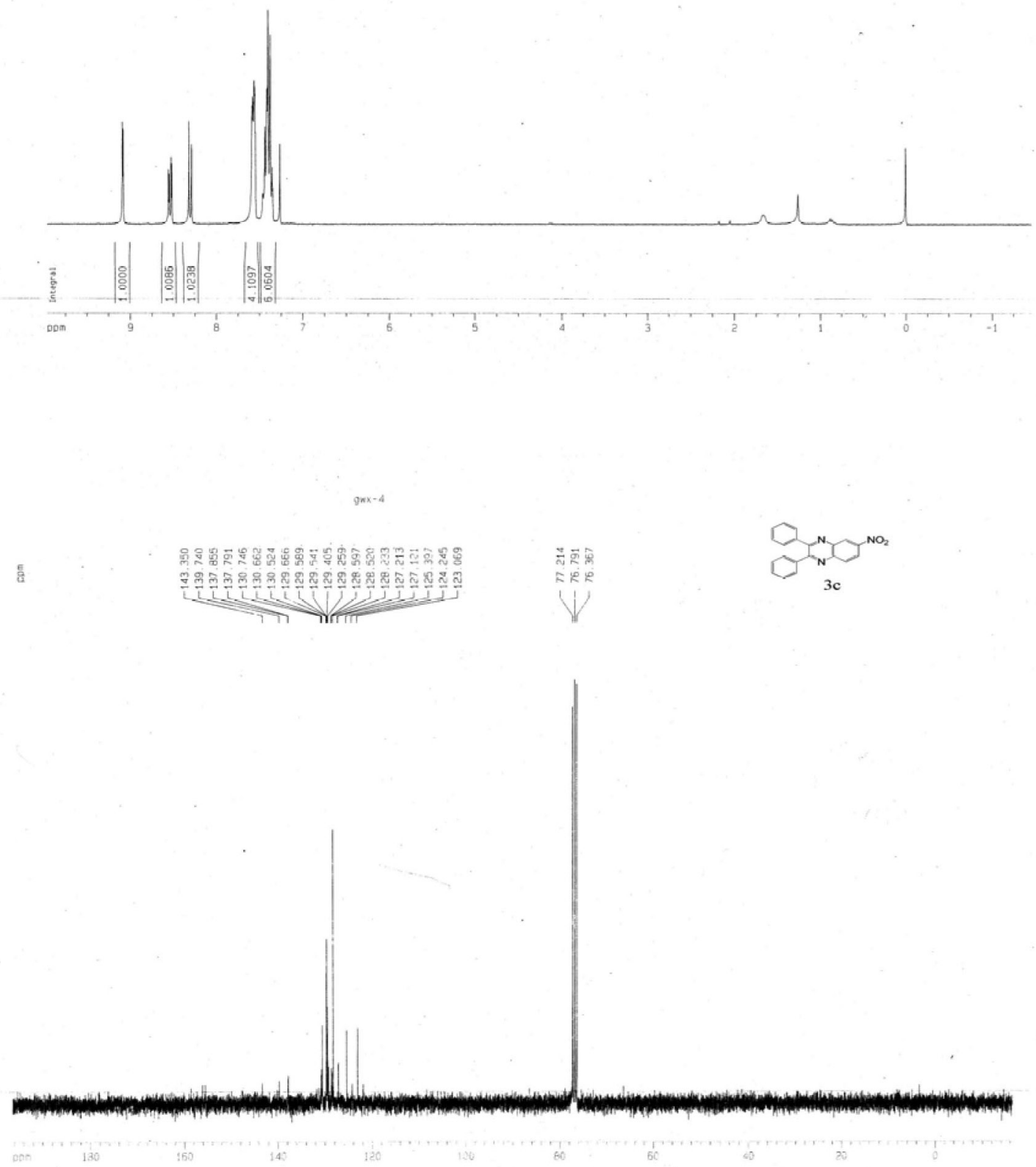

Figure S3. ${ }^{1} \mathrm{H}$ NMR of $\mathbf{3 c}\left(300 \mathrm{MHz}, \mathrm{CDCl}_{3}\right)$ and ${ }^{13} \mathrm{C} \mathrm{NMR}$ of $\mathbf{3 c}\left(75 \mathrm{MHz}, \mathrm{CDCl}_{3}\right)$. 


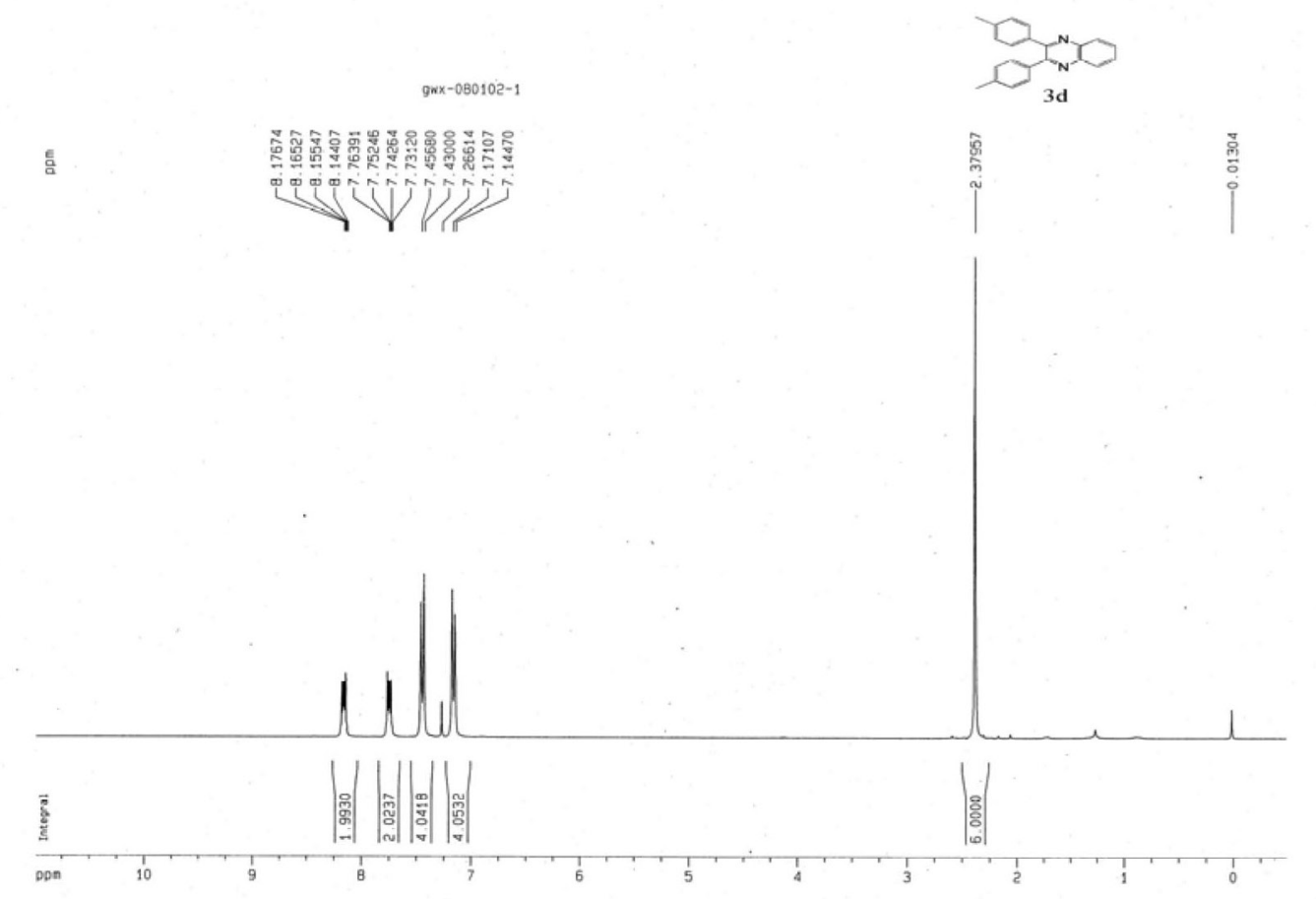

gwx-080102-1
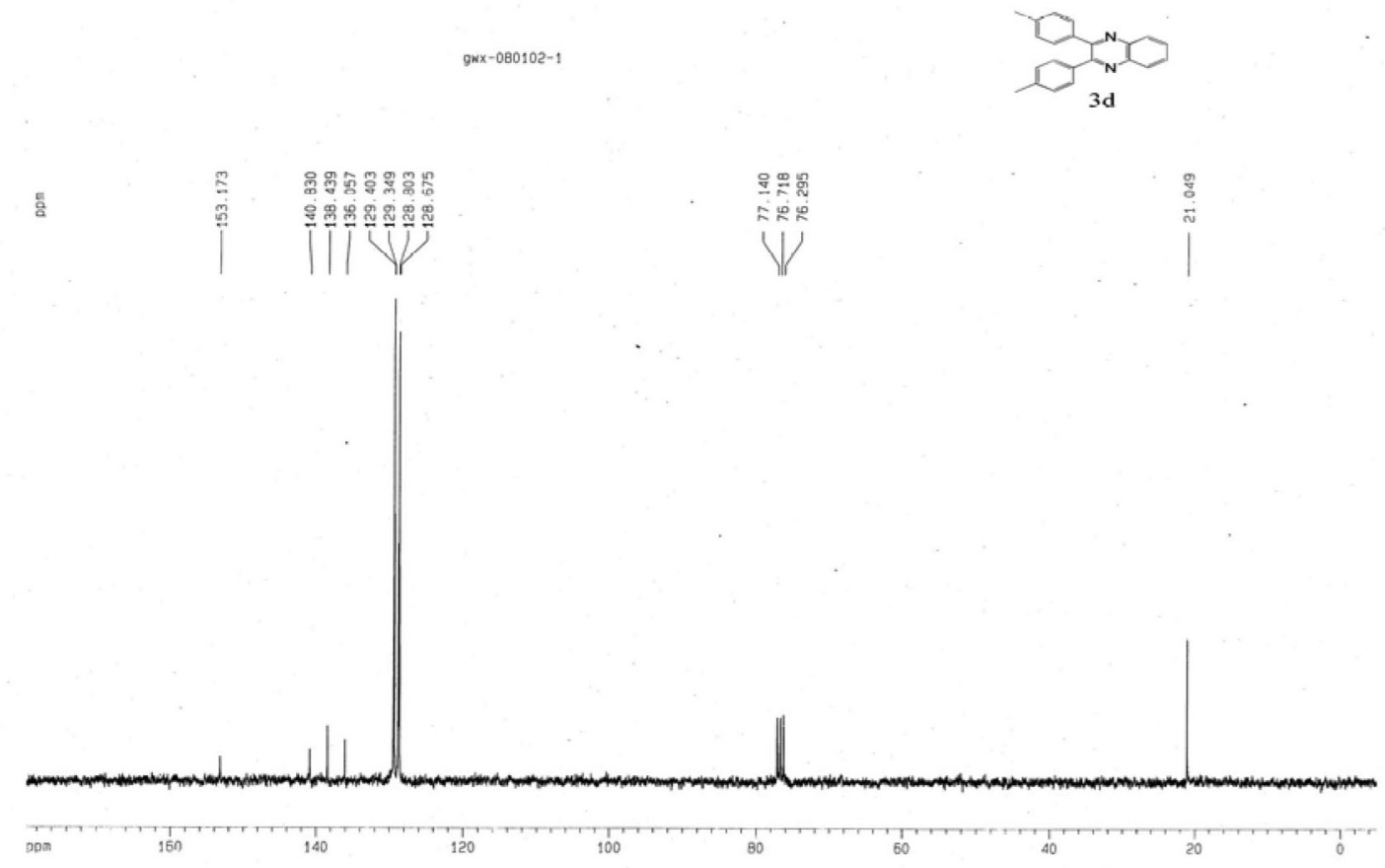

Figure S4. ${ }^{1} \mathrm{H}$ NMR of $\mathbf{3 d}\left(300 \mathrm{MHz}, \mathrm{CDCl}_{3}\right)$ and ${ }^{13} \mathrm{C} \mathrm{NMR}$ of $\mathbf{3 d}\left(75 \mathrm{MHz}, \mathrm{CDCl}_{3}\right)$. 

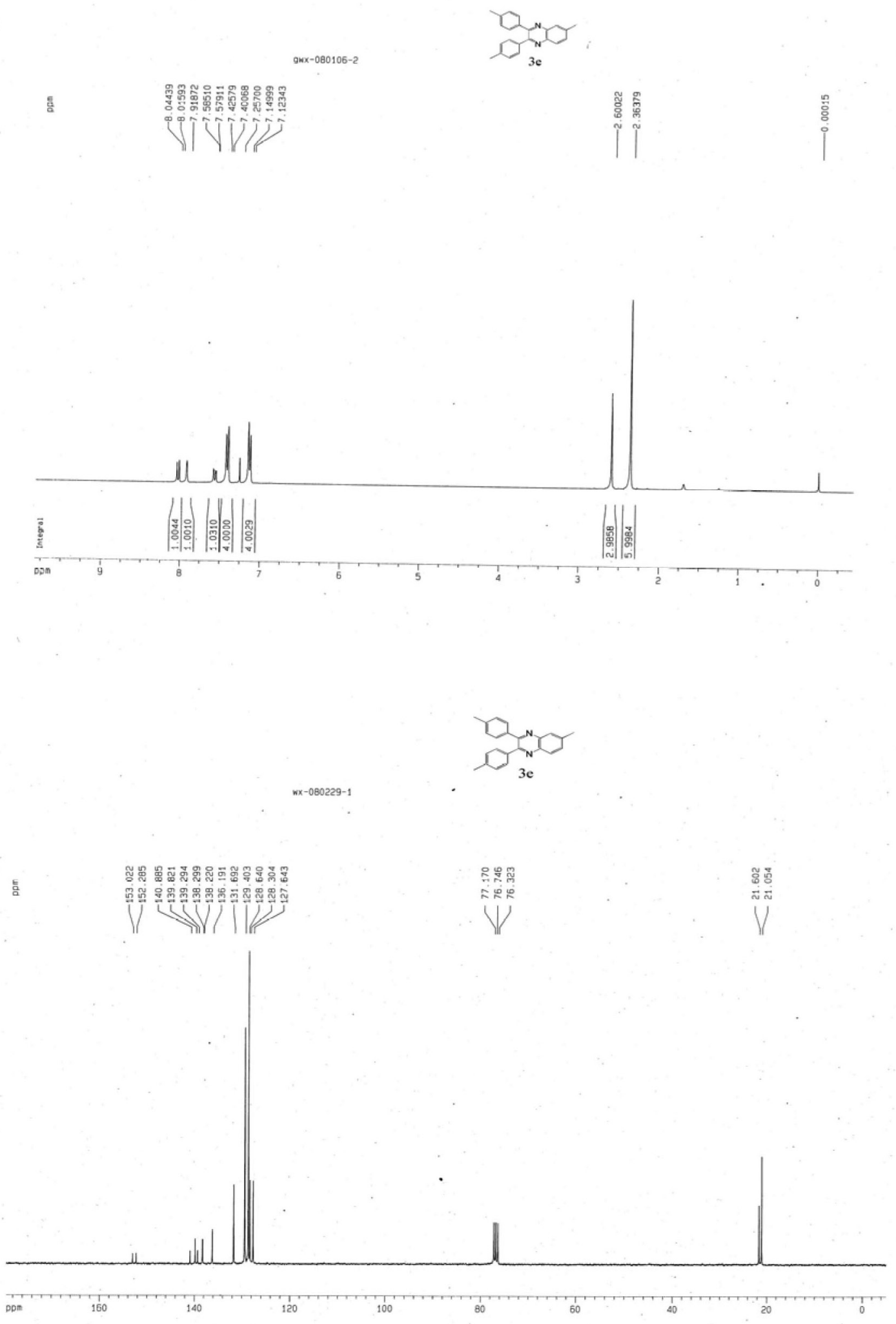

Figure S5. ${ }^{1} \mathrm{H} \mathrm{NMR}$ of $\mathbf{3 e}\left(300 \mathrm{MHz}, \mathrm{CDCl}_{3}\right)$ and ${ }^{13} \mathrm{C} \mathrm{NMR}$ of $\mathbf{3 e}\left(75 \mathrm{MHz}, \mathrm{CDCl}_{3}\right)$. 


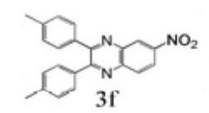

s

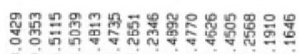

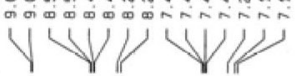
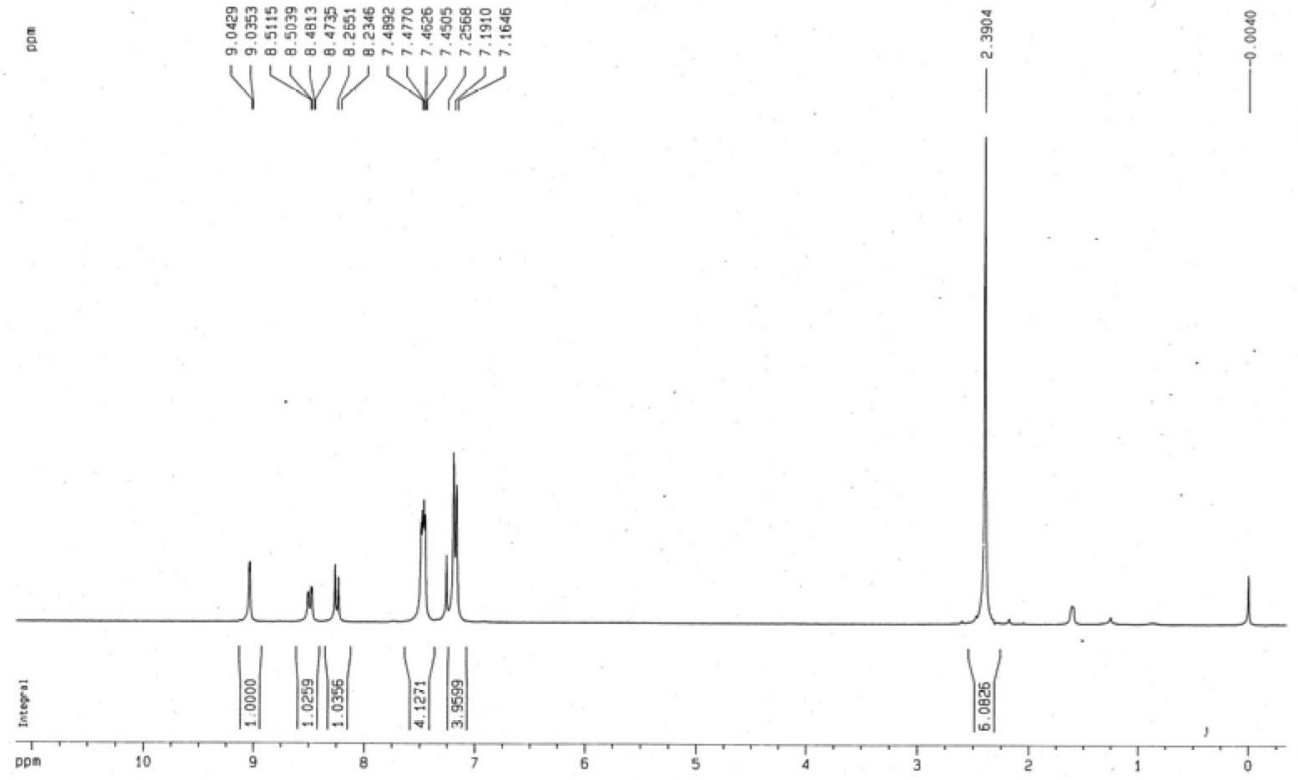

$9 n x-080106-3$
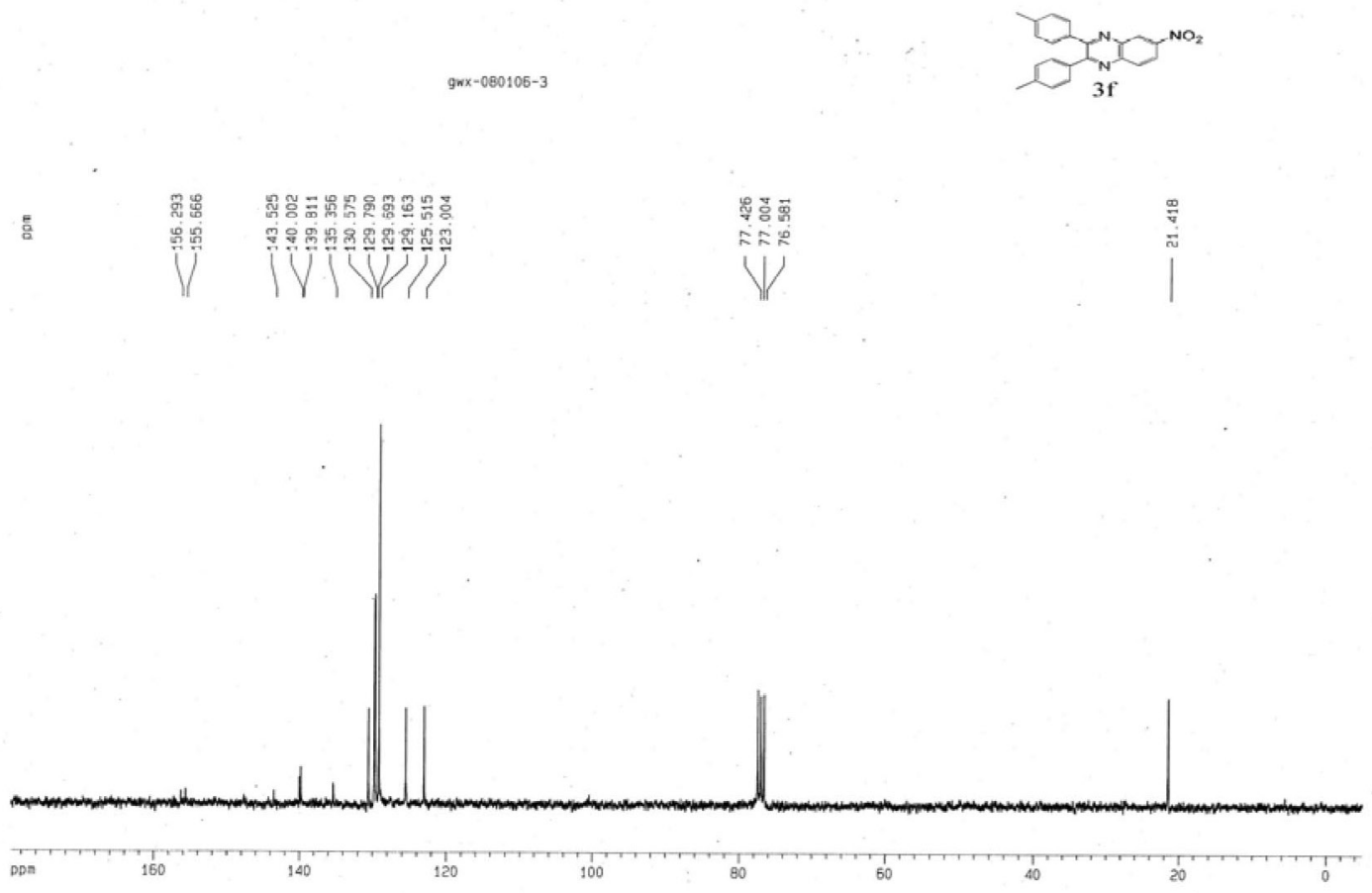

Figure S6. ${ }^{1} \mathrm{H}$ NMR of $\mathbf{3 f}\left(300 \mathrm{MHz}, \mathrm{CDCl}_{3}\right)$ and ${ }^{13} \mathrm{C} \mathrm{NMR}$ of $\mathbf{3 f}\left(75 \mathrm{MHz}, \mathrm{CDCl}_{3}\right)$. 


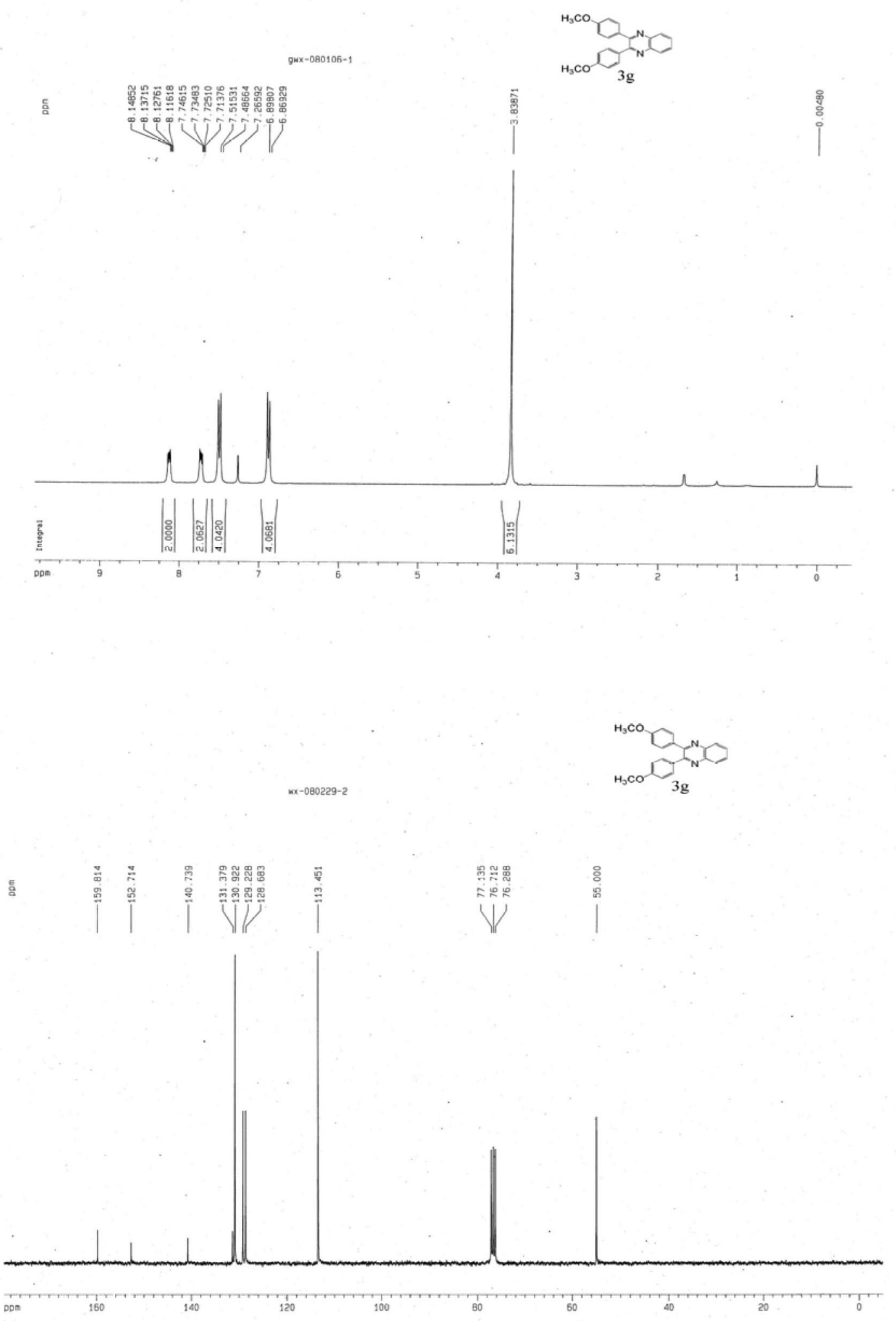

Figure S7. ${ }^{1} \mathrm{H}$ NMR of $\mathbf{3 g}\left(300 \mathrm{MHz}, \mathrm{CDCl}_{3}\right)$ and ${ }^{13} \mathrm{C} \mathrm{NMR}$ of $\mathbf{3 g}\left(75 \mathrm{MHz}, \mathrm{CDCl}_{3}\right)$. 
SP

An Efficient Catalyst-Free Protocol for the Synthesis of Quinoxaline Derivatives

J. Braz. Chem. Soc.
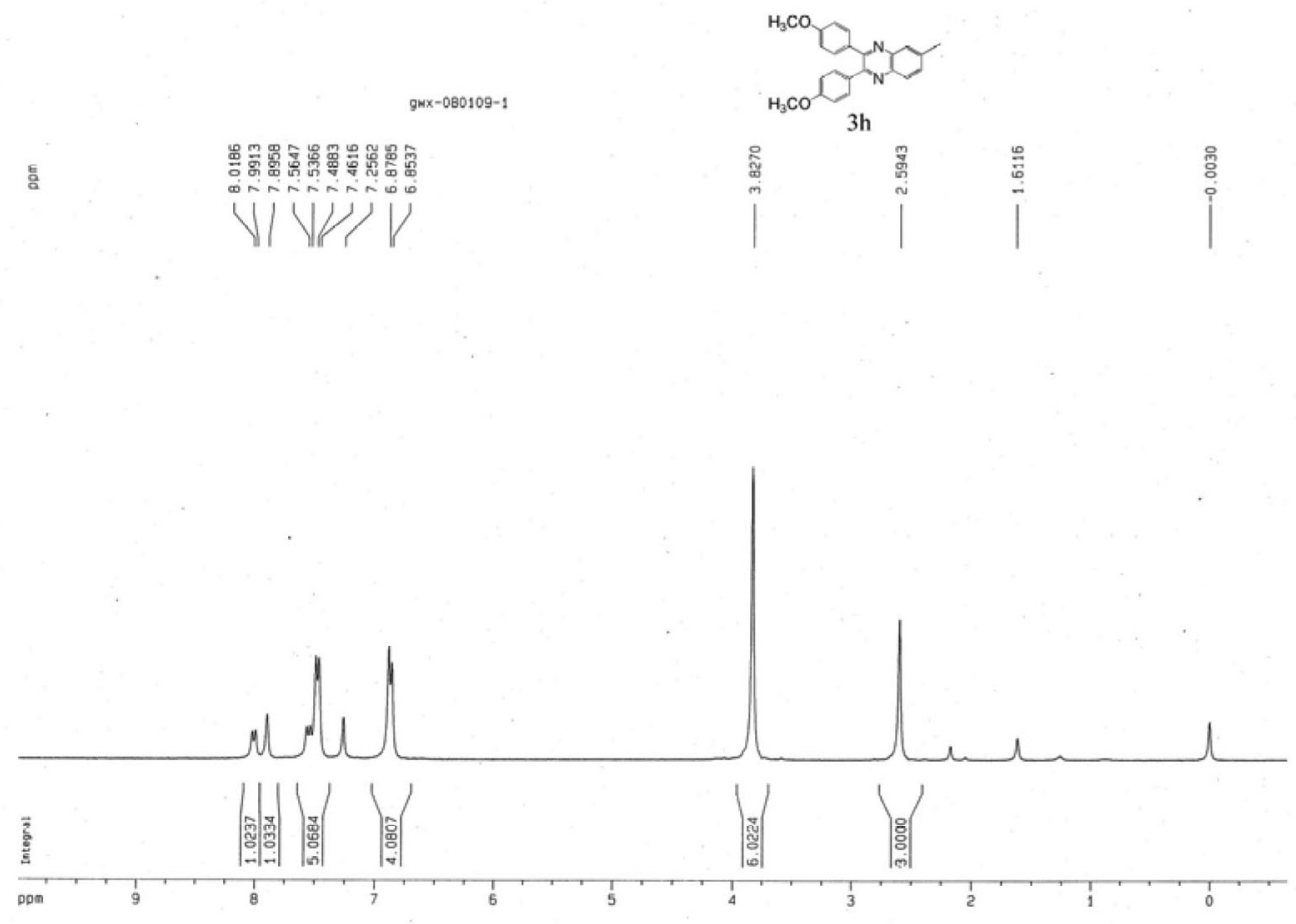

gwx-060109-1
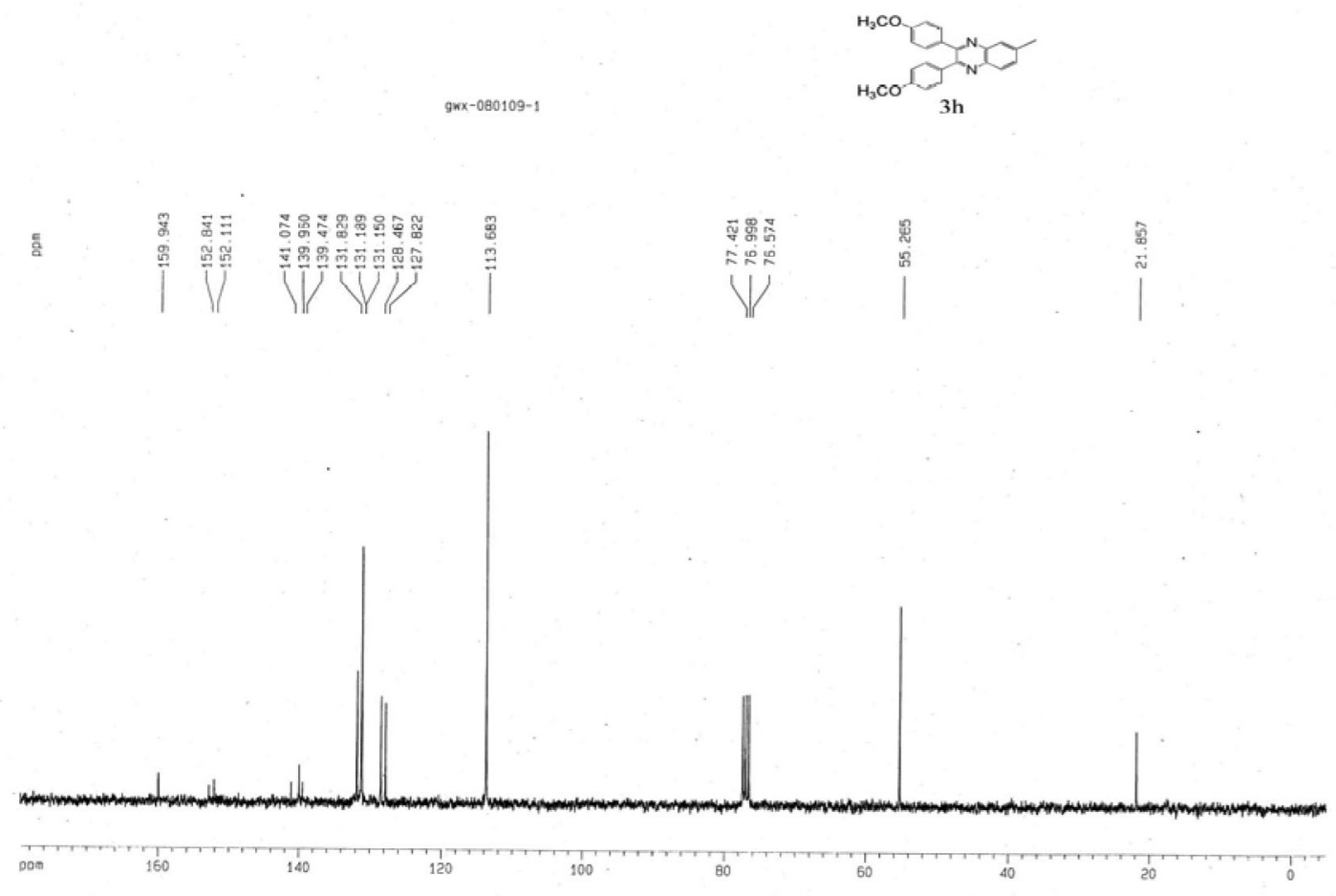

Figure S8. ${ }^{1} \mathrm{H}$ NMR of $\mathbf{3 h}\left(300 \mathrm{MHz}, \mathrm{CDCl}_{3}\right)$ and ${ }^{13} \mathrm{C} \mathrm{NMR}$ of $\mathbf{3 h}\left(75 \mathrm{MHz}, \mathrm{CDCl}_{3}\right)$. 


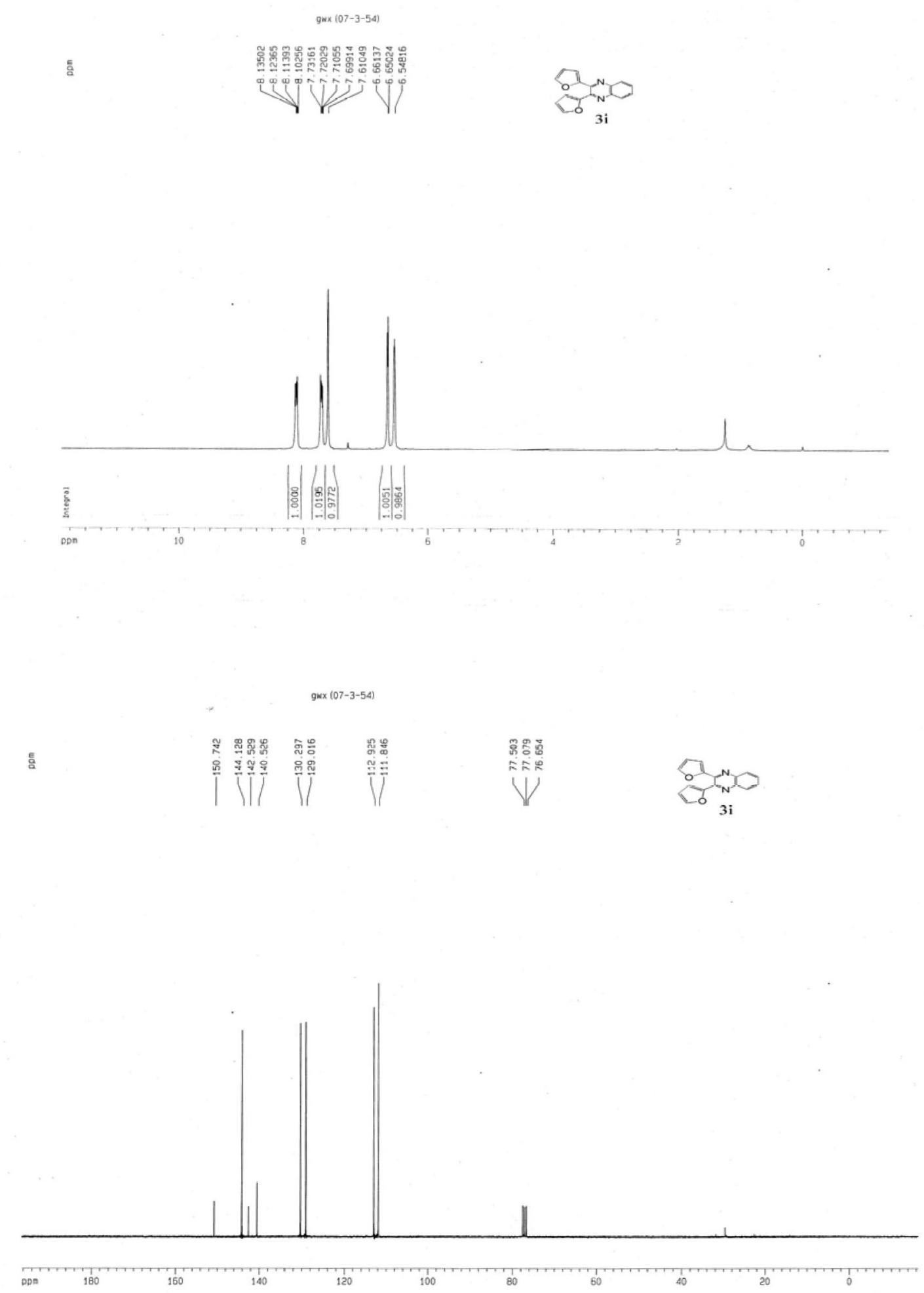

Figure S9. ${ }^{1} \mathrm{H} \mathrm{NMR}$ of $\mathbf{3 i}\left(300 \mathrm{MHz}, \mathrm{CDCl}_{3}\right)$ and ${ }^{13} \mathrm{C} \mathrm{NMR}$ of $\mathbf{3 i}\left(75 \mathrm{MHz}, \mathrm{CDCl}_{3}\right)$. 
gex $x=d$

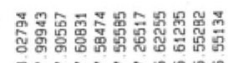

जiञग

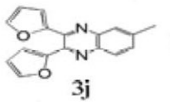

|
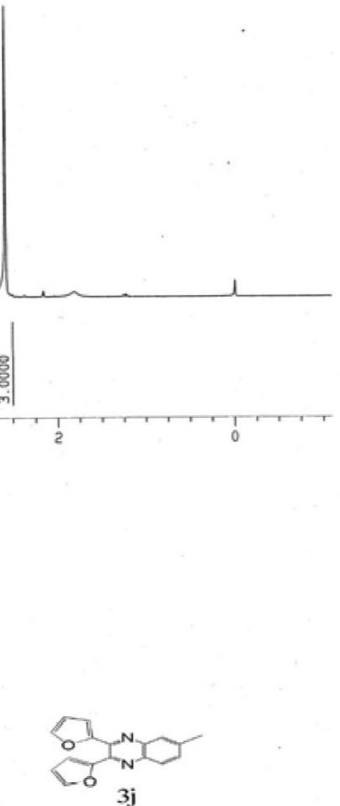

$3 \mathbf{j}$

镸

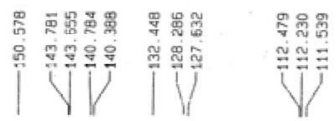
蛋颌
₹果电
(V)
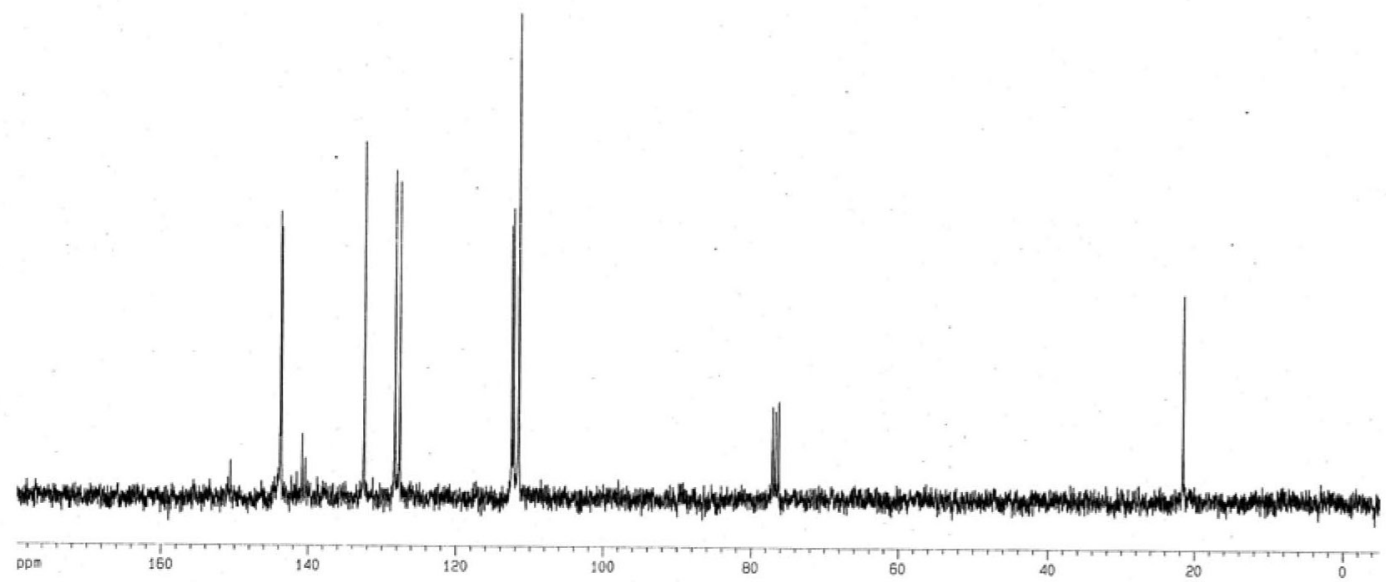

${ }_{60}^{1}$

${ }_{40}^{10}$

20

Figure S10. ${ }^{1} \mathrm{H}$ NMR of $\mathbf{3 j}\left(300 \mathrm{MHz}, \mathrm{CDCl}_{3}\right)$ and ${ }^{13} \mathrm{C} \mathrm{NMR}$ of $\mathbf{3 j}\left(75 \mathrm{MHz}, \mathrm{CDCl}_{3}\right.$ ). 


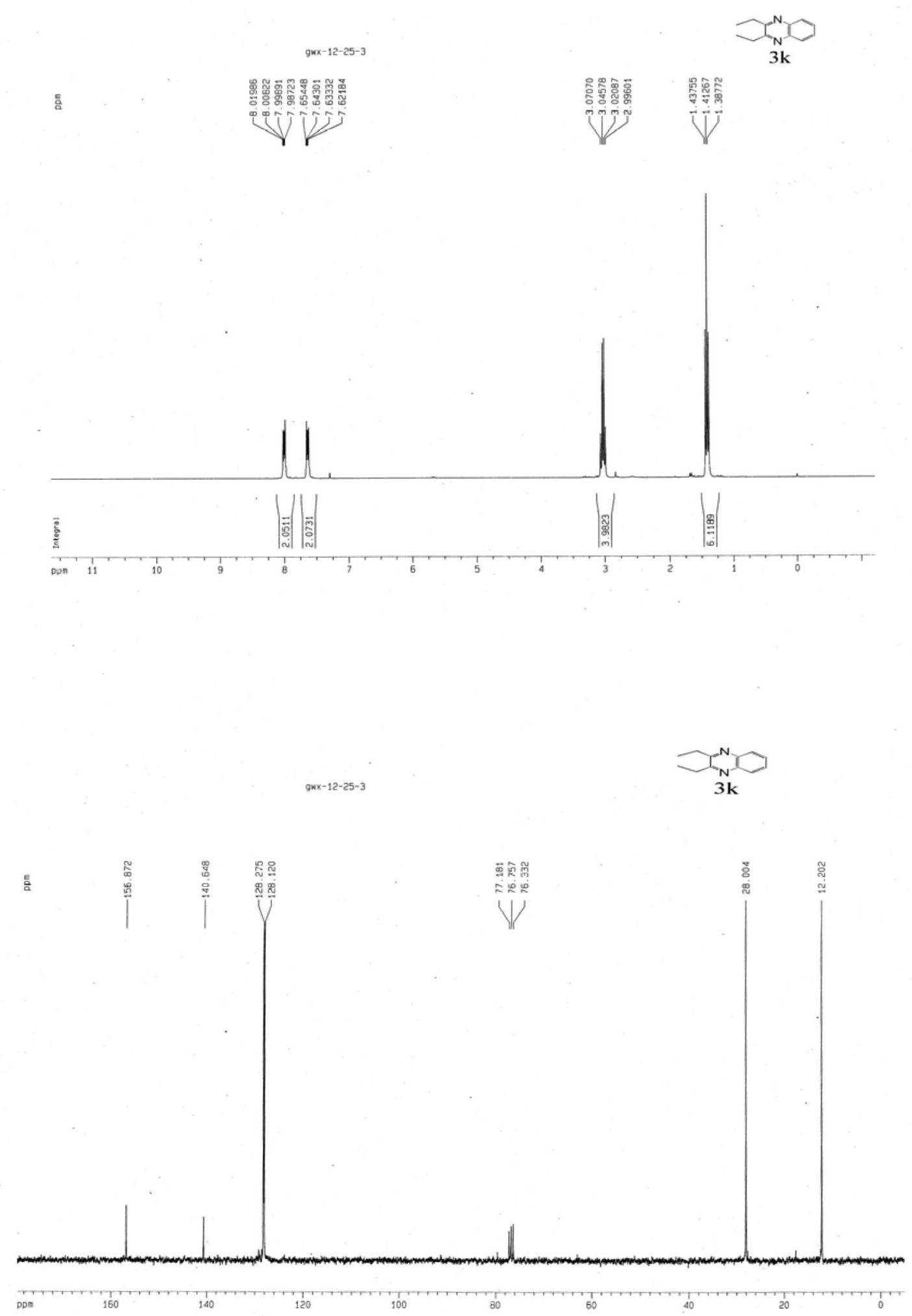

Figure S11. ${ }^{1} \mathrm{H}$ NMR of $\mathbf{3 k}\left(300 \mathrm{MHz}, \mathrm{CDCl}_{3}\right)$ and ${ }^{13} \mathrm{C} \mathrm{NMR}$ of $\mathbf{3 k}\left(75 \mathrm{MHz}, \mathrm{CDCl}_{3}\right)$. 

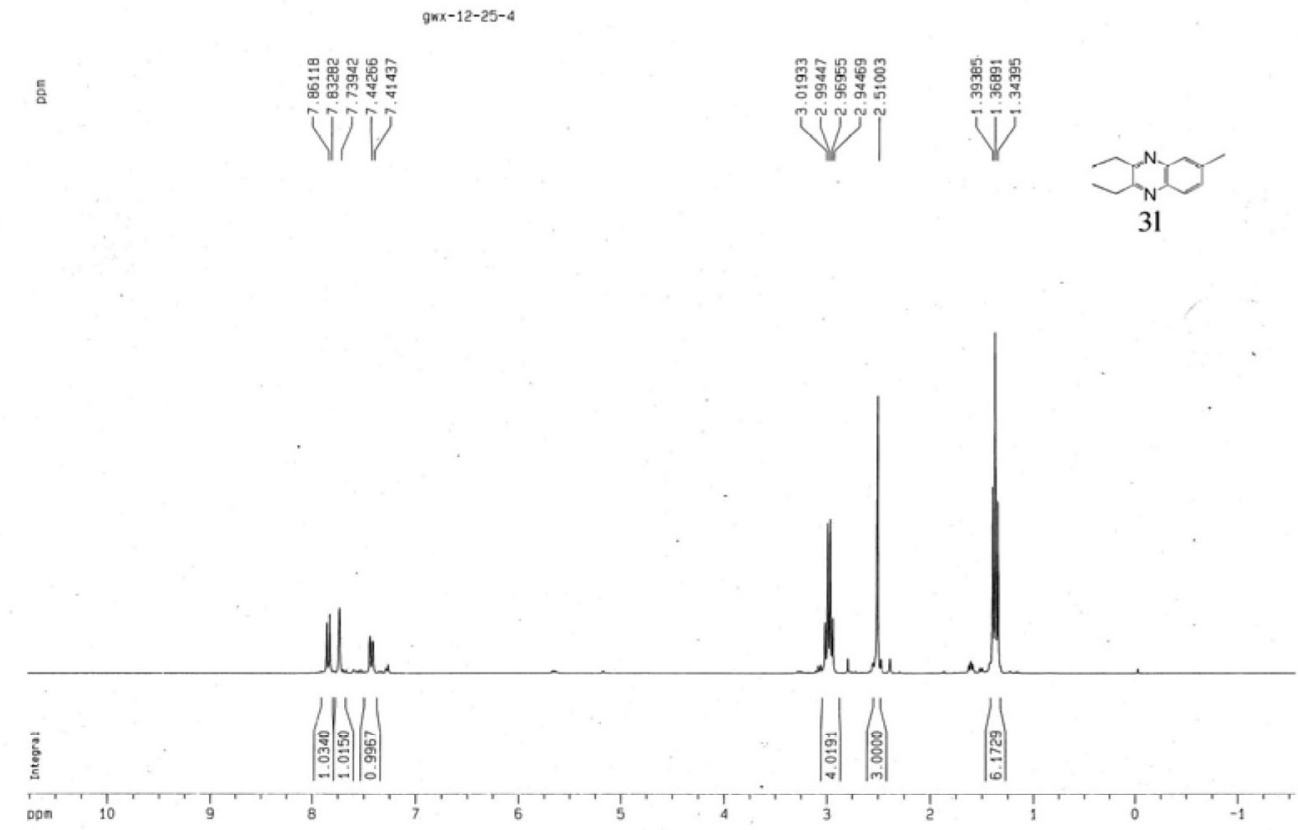

$9 \times x-12-25-4$
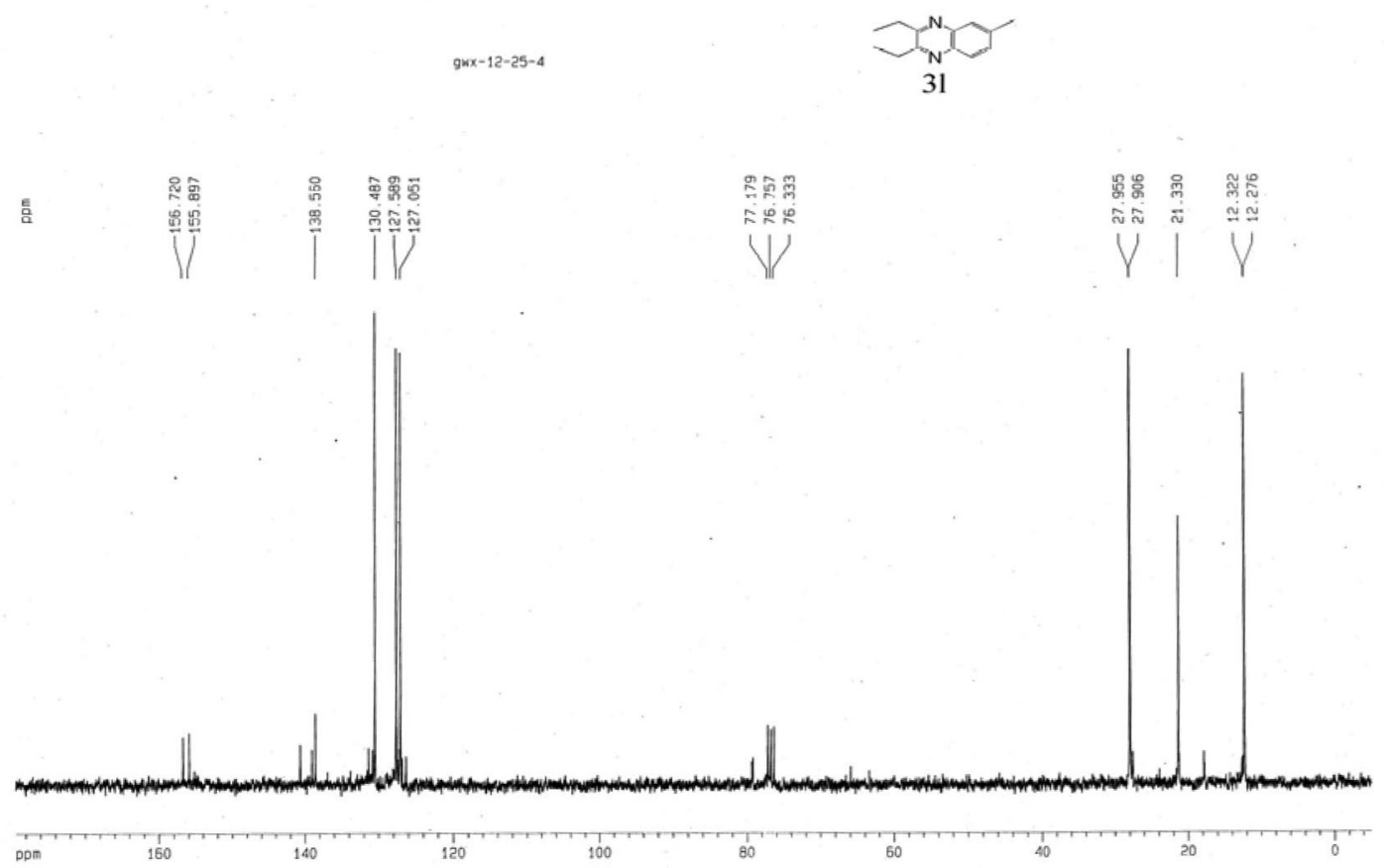

Figure S12. ${ }^{1} \mathrm{H}$ NMR of $\mathbf{3 l}\left(300 \mathrm{MHz}, \mathrm{CDCl}_{3}\right)$ and ${ }^{13} \mathrm{C} \mathrm{NMR}$ of $\mathbf{3 l}\left(75 \mathrm{MHz}, \mathrm{CDCl}_{3}\right)$. 


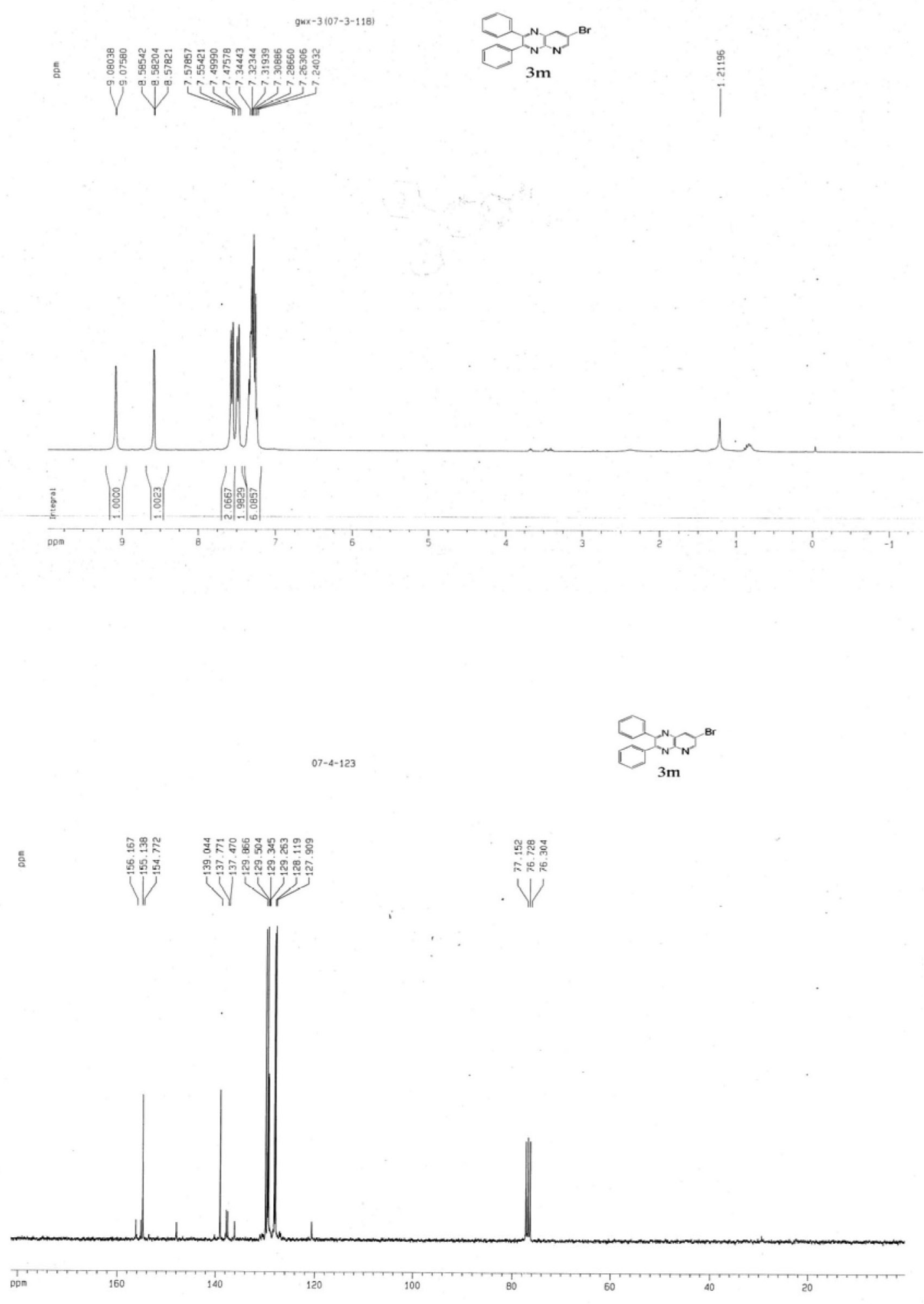

Figure S13. ${ }^{1} \mathrm{H}$ NMR of $\mathbf{3 m}\left(300 \mathrm{MHz}, \mathrm{CDCl}_{3}\right)$ and ${ }^{13} \mathrm{C} \mathrm{NMR}$ of $\mathbf{3 m}\left(75 \mathrm{MHz}, \mathrm{CDCl}_{3}\right)$. 
$9 \times x-080109-3$
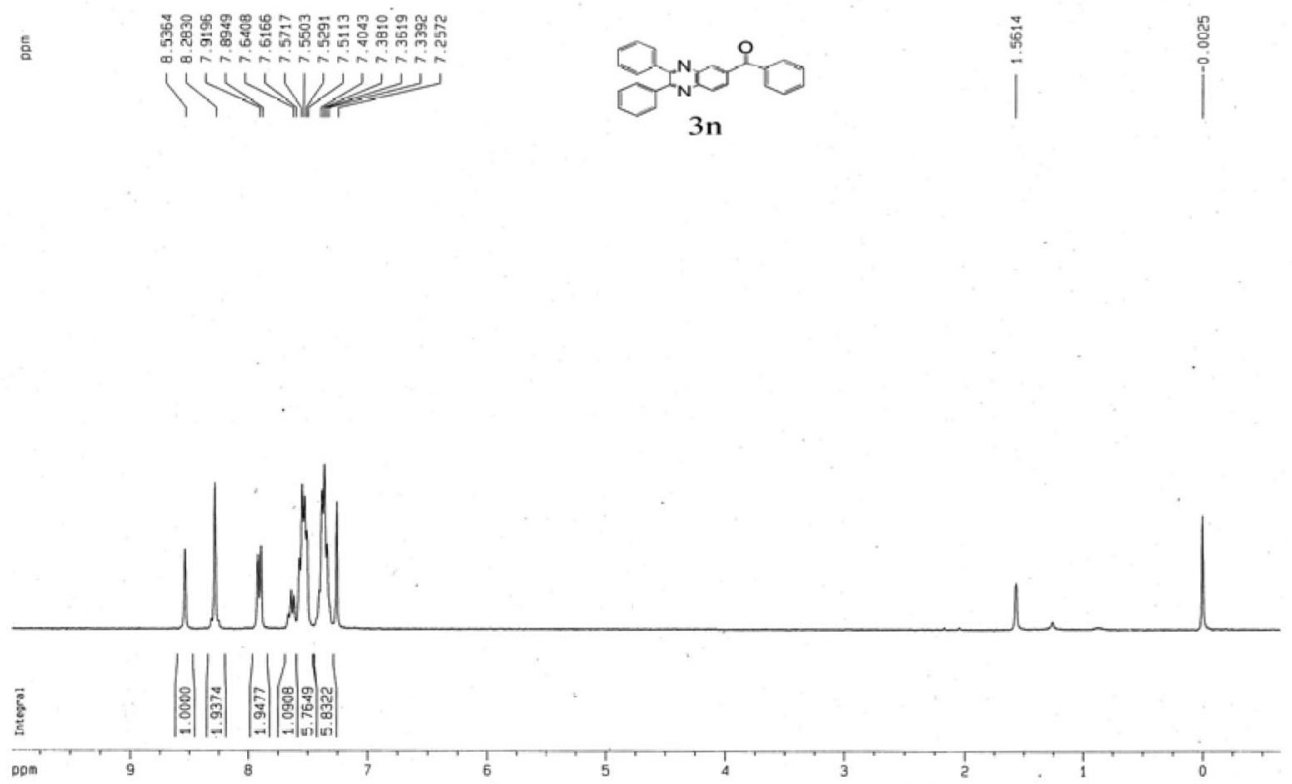

$9 * x-080109-3$
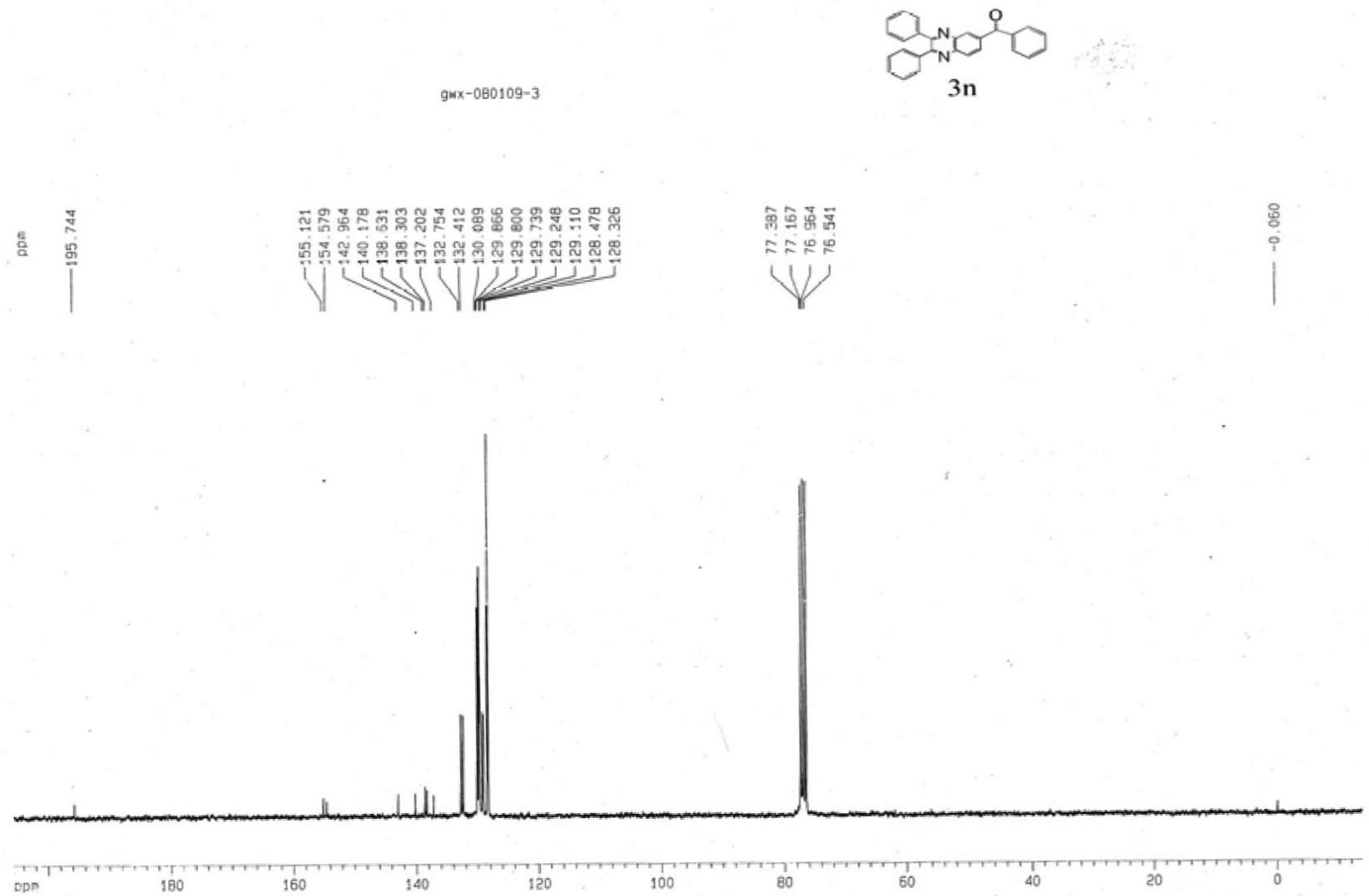

Figure S14. ${ }^{1} \mathrm{H}$ NMR of $3 \mathbf{n}\left(300 \mathrm{MHz}, \mathrm{CDCl}_{3}\right)$ and ${ }^{13} \mathrm{C} \mathrm{NMR}$ of $\mathbf{3 n}\left(75 \mathrm{MHz}, \mathrm{CDCl}_{3}\right)$. 
Vol. 20, No. 9, 2009

Gus et al.

S15

$07-4-122$

$\mathrm{a}$
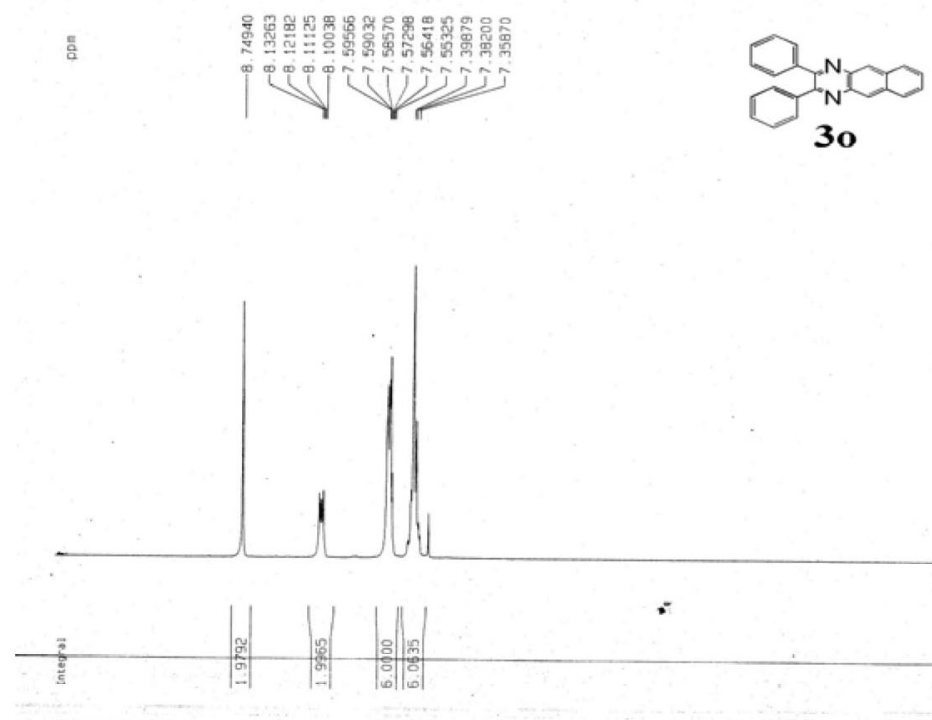

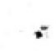

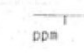

$07-4-122$
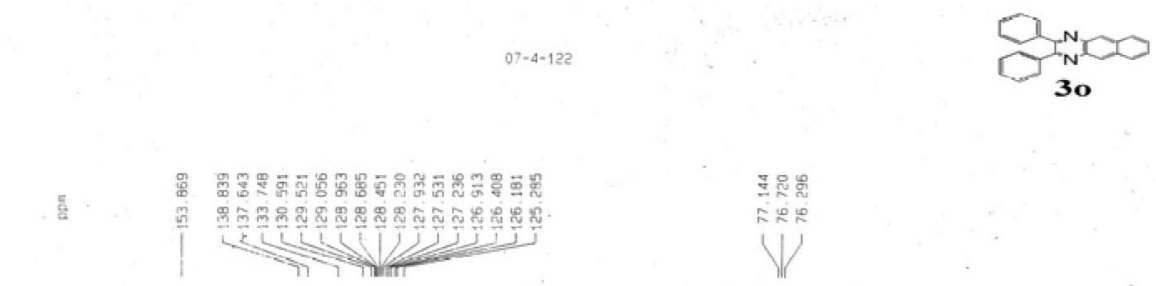

Figure S15. ${ }^{1} \mathrm{H}$ NMR of $\mathbf{3 o}\left(300 \mathrm{MHz}, \mathrm{CDCl}_{3}\right)$ and ${ }^{13} \mathrm{C} \mathrm{NMR}$ of $\mathbf{3 o}\left(75 \mathrm{MHz}, \mathrm{CDCl}_{3}\right)$.

। 


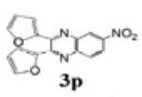

镸

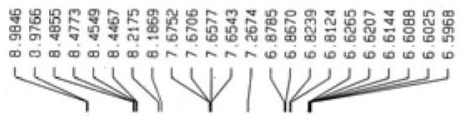

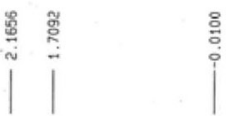

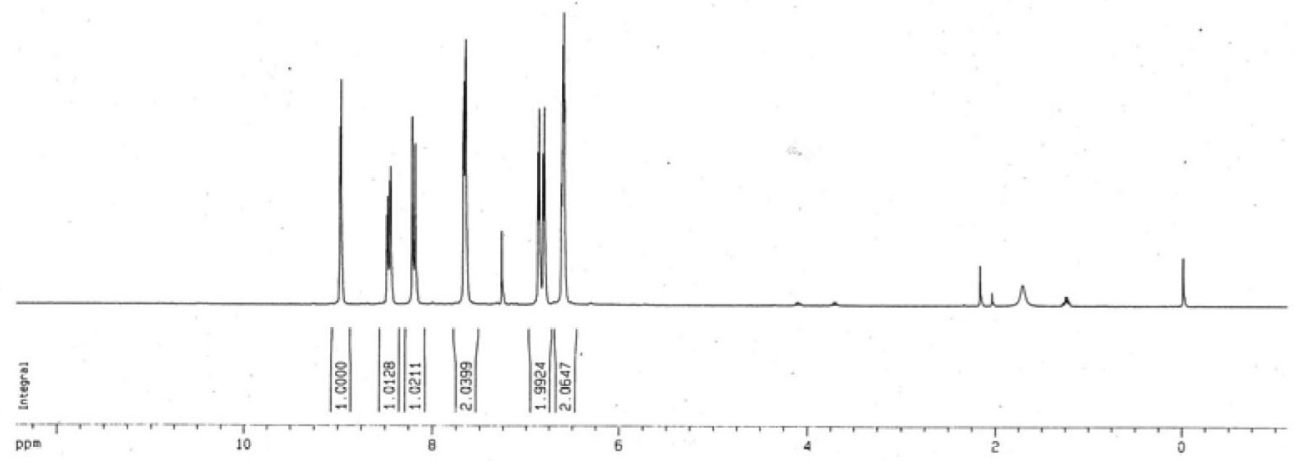

$g n x=c$
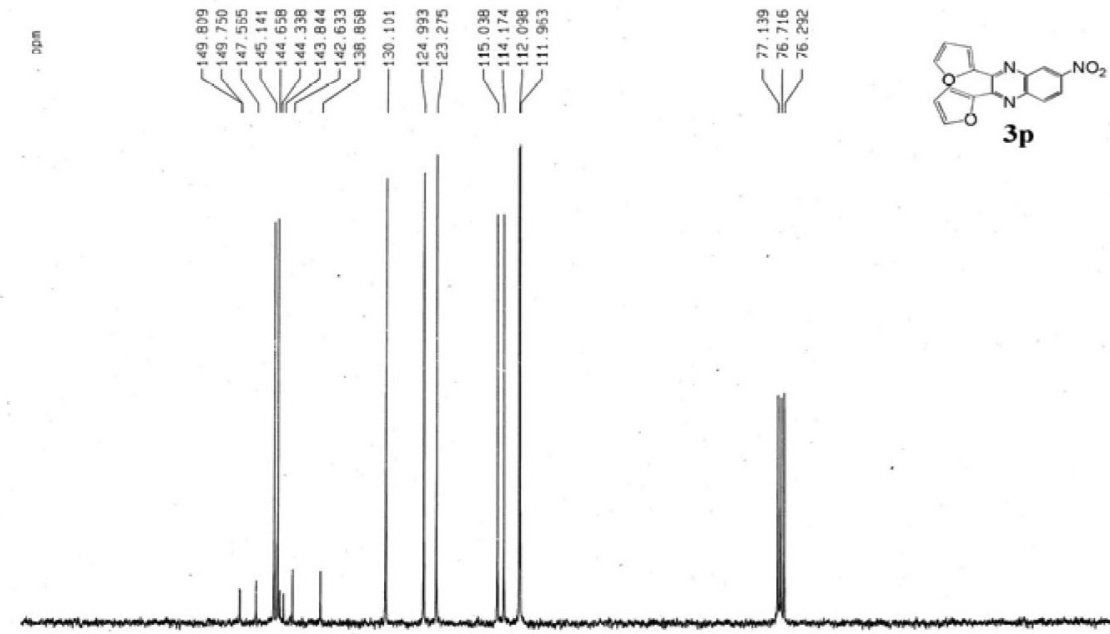

$3 p$

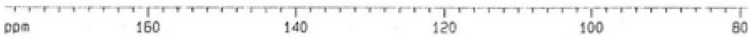

80

${ }^{1} 0^{\prime}$

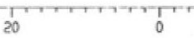

Figure S16. ${ }^{1} \mathrm{H}$ NMR of $\mathbf{3 p}\left(300 \mathrm{MHz}, \mathrm{CDCl}_{3}\right)$ and ${ }^{13} \mathrm{C} \mathrm{NMR}$ of $\mathbf{3 p}\left(75 \mathrm{MHz}, \mathrm{CDCl}_{3}\right)$. 
$g n x-6$

⿷

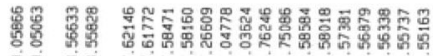

iv vicivivipos

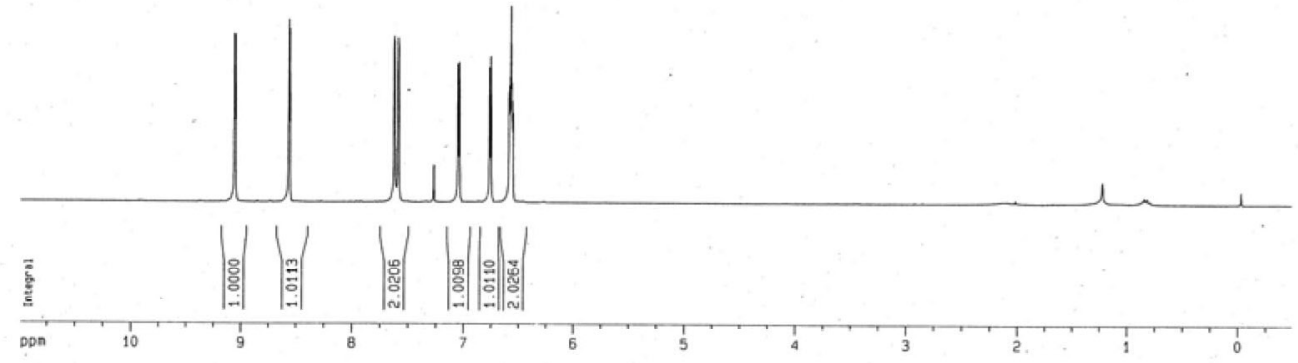

$g n x x-6$

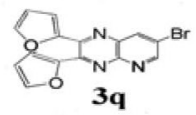

镸

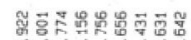

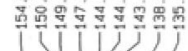

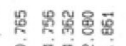

율

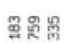

₹श:

(V)

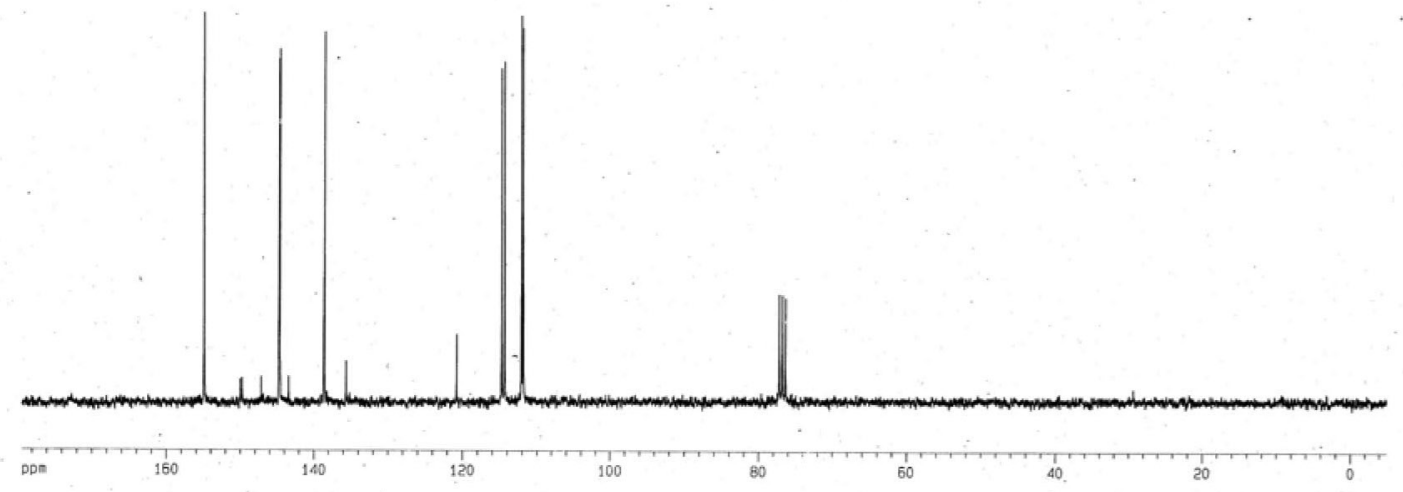

Figure S17. ${ }^{1} \mathrm{H}$ NMR of $\mathbf{3 q}\left(300 \mathrm{MHz}, \mathrm{CDCl}_{3}\right)$ and ${ }^{13} \mathrm{C} \mathrm{NMR}$ of $\mathbf{3 q}\left(75 \mathrm{MHz}, \mathrm{CDCl}_{3}\right)$. 
$\operatorname{gnx} x-12-25-1$
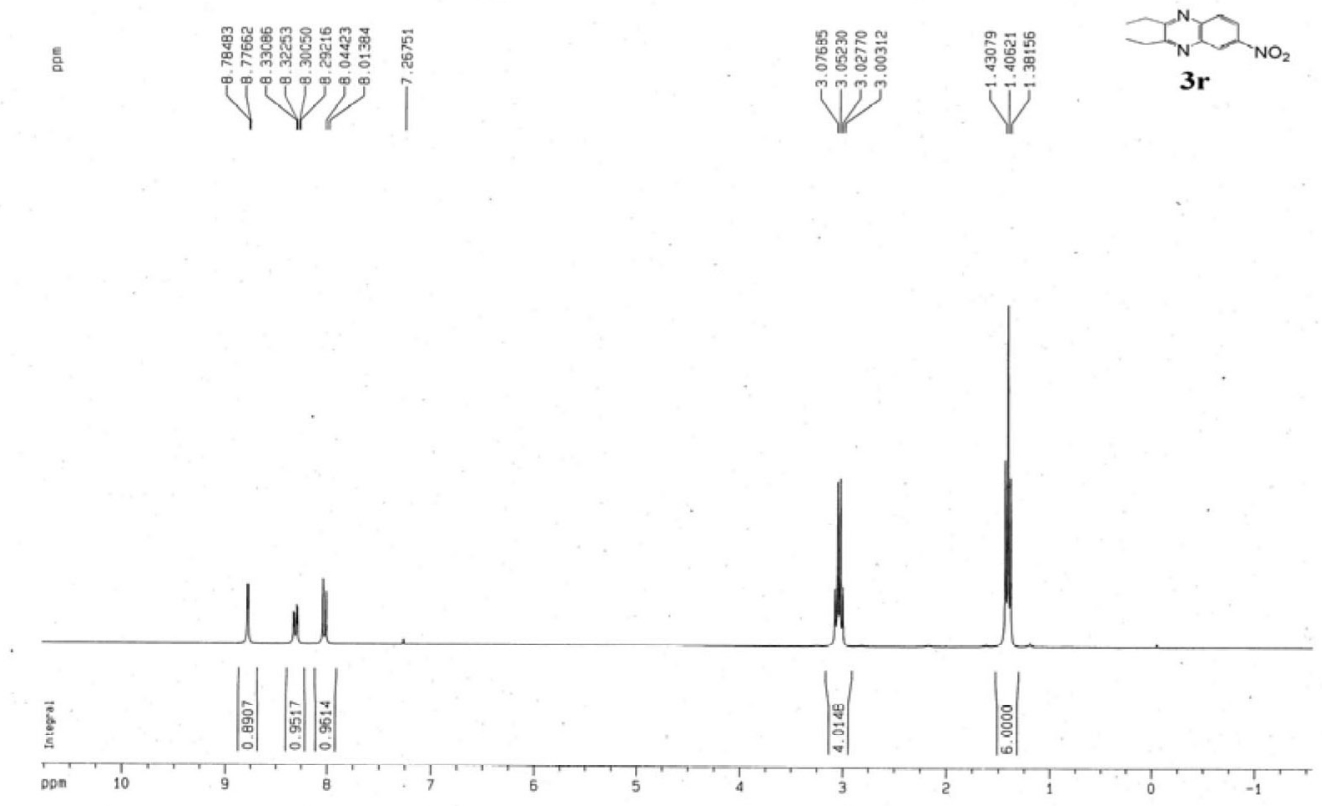

gwx-12-25- 1
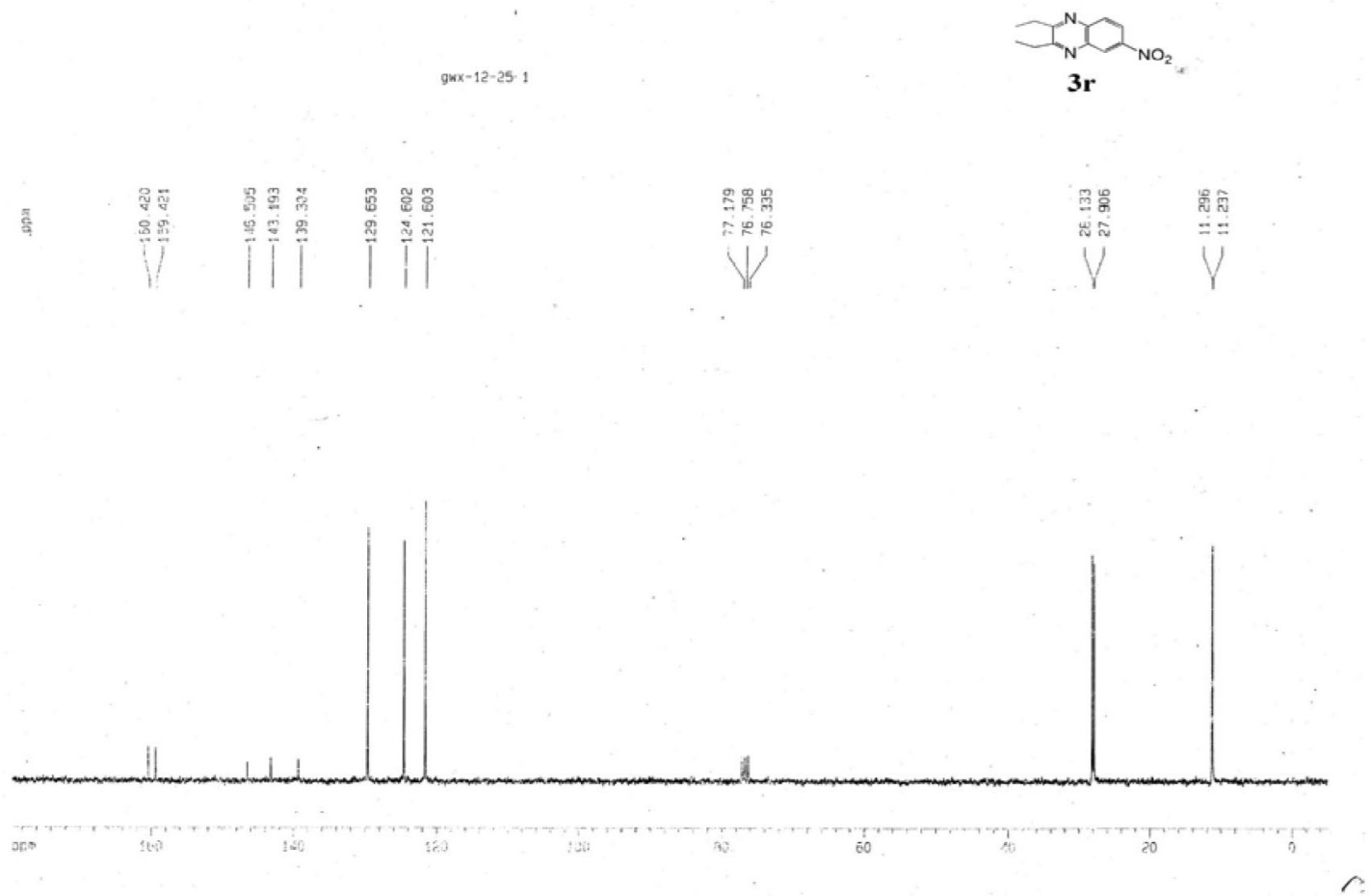

Figure S18. ${ }^{1} \mathrm{H}$ NMR of $\mathbf{3 r}\left(300 \mathrm{MHz}, \mathrm{CDCl}_{3}\right)$ and ${ }^{13} \mathrm{C} \mathrm{NMR}$ of $\mathbf{3 r}\left(75 \mathrm{MHz}, \mathrm{CDCl}_{3}\right)$. 
$20 x-12-25-2$

รัฐ
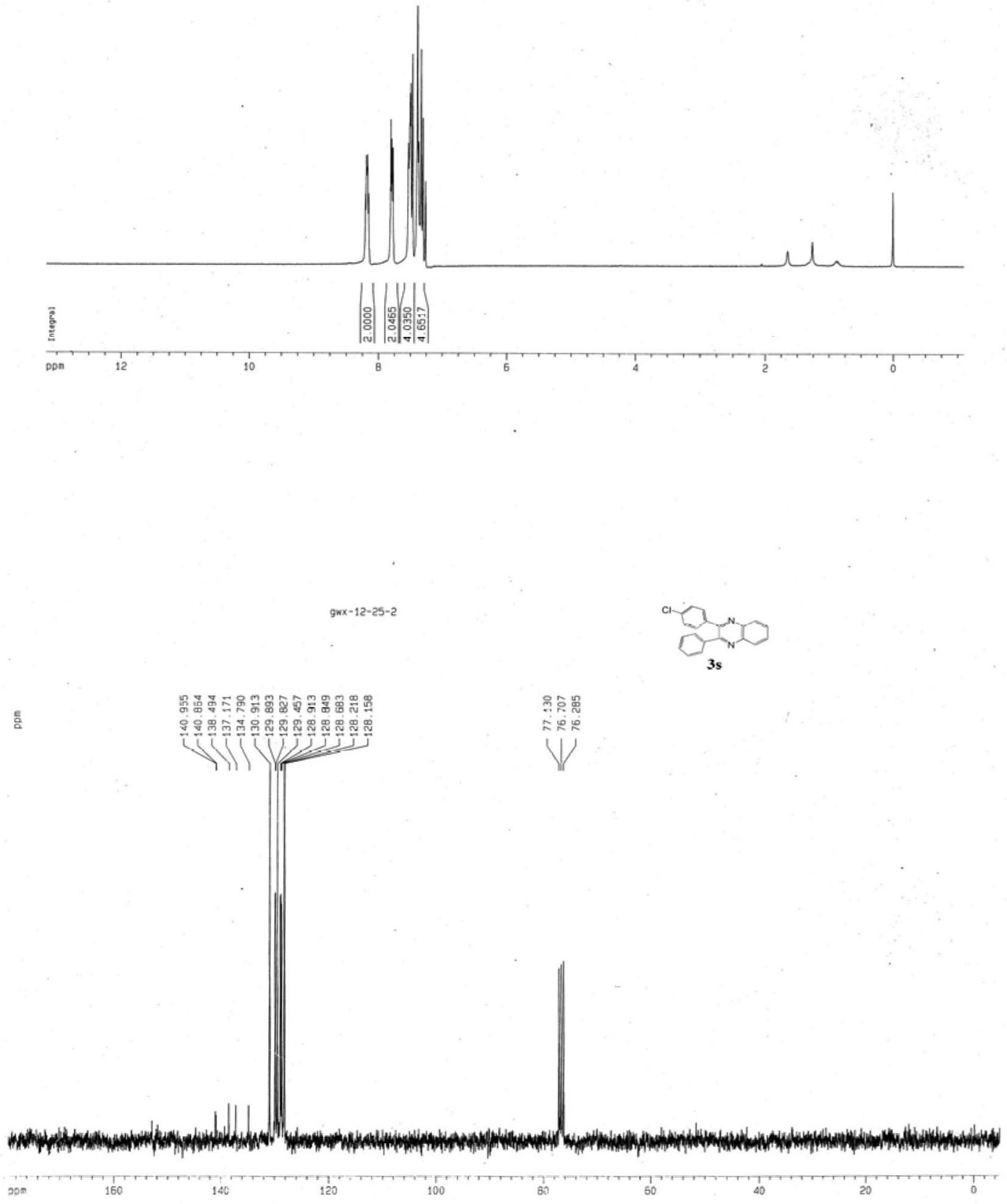

Figure S19. ${ }^{1} \mathrm{H}$ NMR of $\mathbf{3 s}\left(300 \mathrm{MHz}, \mathrm{CDCl}_{3}\right)$ and ${ }^{13} \mathrm{C} \mathrm{NMR}$ of $\mathbf{3 s}\left(75 \mathrm{MHz}, \mathrm{CDCl}_{3}\right)$. 